\title{
ON THE LEBESGUE MEASURE OF LI-YORKE PAIRS FOR INTERVAL MAPS
}

\author{
HENK BRUIN AND VÍCTOR JIMÉNEZ LÓPEZ
}

\begin{abstract}
We investigate the prevalence of Li-Yorke pairs for $C^{2}$ and $C^{3}$ multimodal maps $f$ with non-flat critical points. We show that every measurable scrambled set has zero Lebesgue measure and that all strongly wandering sets have zero Lebesgue measure, as does the set of pairs of asymptotic (but not asymptotically periodic) points.

If $f$ is topologically mixing and has no Cantor attractor, then typical (w.r.t. twodimensional Lebesgue measure) pairs are Li-Yorke; if additionally $f$ admits an absolutely continuous invariant probability measure (acip), then typical pairs have a dense orbit for $f \times f$. These results make use of so-called nice neighborhoods of the critical set of general multimodal maps, and hence uniformly expanding Markov induced maps, the existence of either is proved in this paper as well.

For the setting where $f$ has a Cantor attractor, we present a trichotomy explaining when the set of Li-Yorke pairs and distal pairs have positive two-dimensional Lebesgue measure.
\end{abstract}

\section{INTRODUCTION}

In interval dynamics there are many ways to deal with the notion of asymptotic complexity ("chaos"). Probably it is pointless to try and decide which is the best of them, but in applications there are two of them which are by far the most popular, see e.g. [D, [MMN]. One is topological chaos, that is, the existence of an uncountable scrambled set in the sense of the famous Li and Yorke paper [LY]. The other one is ergodic chaos, that is, the existence of an invariant probability measure absolutely continuous with respect to the Lebesgue measure (acip). Neither of them is without drawbacks. (To keep this introduction at the expository level, we have deferred most of definitions to the subsequent sections.)

There are easy conditions implying the existence of Li-Yorke chaos and its stability under small perturbations. One such condition is the existence of a periodic orbit of period not a power of two [B]]. Nevertheless, this chaos need not be "observable": for instance, the

Date: November 2, 2018.

2000 Mathematics Subject Classification. Primary: 37E05, Secondary: 26A18, 37B20, 37C70.

Key words and phrases. Li-Yorke pair, Li-Yorke chaos, scrambled set, interval map, distal pair, asymptotic pair, attractor, strongly wandering set.

HB gratefully acknowledges the support of EPSRC grant EP/F037112/1 and also the hospitality of the University of Murcia and Delft University of Technology.

VJL was partially supported by MICINN (Ministerio de Ciencia e Innovacion) and FEDER (Fondo Europeo de Desarrollo Regional), grant MTM2008-03679/MTM, and Fundación Séneca (Agencia de Ciencia y Tecnología de la Región de Murcia, Programa de Generación de Conocimiento Científico de Excelencia, II PCTRM 2007-10), grant 08667/PI/08. 
orbits of almost all points (in the sense of Lebesgue measure) may be attracted by this periodic orbit $[\mathrm{Gu}$.

On the other hand, ergodic chaos ensures complicated dynamics for a set of points with positive measure. For instance, if a smooth multimodal map (with non-flat critical points) $f$ has an acip, then, as a simple consequence of the zero measure of Cantor metric attractors [SV] and Proposition 9 below, there is a positive measure set of points whose orbit under $f$ is dense in some interval. However, the converse is not true, even for the family of logistic maps [Jo, Ly2], so acips can only exist under additional conditions (for instance, hyperbolic repelling periodic points and $\left|D f^{n}(f(c))\right| \rightarrow \infty$ for any critical point $c$ of the map $f$ [BRSS] $)$.

A map $f$ is infinitely renormalizable if there is an infinite collection of nested cycles of periodic intervals; the intersection of these cycles is a Cantor set, called a solenoidal attractor (because the suspension over this attractor is a topological solenoid). The Feigenbaum map, or more correctly Coullet-Tresser-Feigenbaum, (see e.g. [MS, pp. 151152]) is the best known example of this. A solenoidal attractor is Lyapunov stable and as shown in [BrJ] (see Proposition 31 below), points in the basin of such an attractor are approximately periodic:

Definition 1. A point $x$ is approximately periodic if for every $\varepsilon>0$ there is a periodic point $p$ such that $\limsup _{n \rightarrow \infty}\left|f^{n}(x)-f^{n}(p)\right|<\varepsilon$.

Hence, up to small errors, almost all points eventually behave as periodic points.

Remark 2. An intrinsic characterization of adding machines (namely any system in which every point is regularly recurrent) is presented in [BK]. In our setting, it applies to the solenoidal attractor itself, whereas approximate periodicity gives information on a neighborhood of the solenoidal attractor.

However, a multimodal (even polynomial) map may have a dense orbit while, simultaneously, almost all orbits are attracted by a Cantor set (a so-called wild attractor) [BKNS]. As we will explain below, in such a case it is still possible but not necessary that a.e. point is approximately periodic.

We see that there is a variety of smooth multimodal maps featuring a certain degree of "observable" dynamical complexity which is, however, not strong enough to be realized by an acip. It is natural to return to the Li-Yorke notion of chaos and investigate to what extent it can be used to measure this complexity. This is what we intend in the present paper.

Definition 3. Let $f: I=[0,1] \rightarrow I$ be a continuous map. A pair of points $(x, y)$ is called:

- distal if $\liminf _{n \rightarrow \infty}\left|f^{n}(y)-f^{n}(x)\right|>0$;

- asymptotic if $\lim _{n \rightarrow \infty}\left|f^{n}(y)-f^{n}(x)\right|=0$;

- Li-Yorke if it is neither asymptotic nor distal, that is,

$$
0=\liminf _{n \rightarrow \infty}\left|f^{n}(y)-f^{n}(x)\right|<\limsup _{n \rightarrow \infty}\left|f^{n}(y)-f^{n}(x)\right| .
$$


We denote the set of distal, asymptotic and Li-Yorke pairs by Dis, Asymp and LY, respectively. A pair that is not distal (hence asymptotic or Li-Yorke) is called proximal. The set of Li-Yorke pairs with $\limsup _{n \rightarrow \infty}\left|f^{n}(y)-f^{n}(x)\right| \geq \varepsilon$ is denoted as $\mathrm{LY}_{\varepsilon}$.

Note that $f^{n}(x) \neq f^{n}(y)$ for all $n \geq 0$ whenever $(x, y)$ is a Li-Yorke or distal pair.

Definition 4. Let $f: I \rightarrow I$ be a continuous map.

- A set $S \subset X$ is called scrambled if any two distinct points in S form a Li-Yorke pair.

- The map $f$ is called chaotic (in the sense of Li-Yorke) if it has an uncountable scrambled set.

The above definitions can be strengthened by assuming that there is a positive lower bound of $\lim \sup \left|f^{n}(x)-f^{n}(y)\right|$ independently of $x, y$ :

- If there is $\varepsilon>0$ such that for every $x \neq y \in S$, $\lim \sup \left|f^{n}(x)-f^{n}(y)\right| \geq \varepsilon$ independent of $x, y \in S$, then $S$ is called $\varepsilon$-scrambled.

- If there is $\varepsilon>0$ such that for every $x \in X$ and every neighborhood $U \ni x$, there is $y \in U$ such that $(x, y) \in \mathrm{LY}_{\varepsilon}$, then $f$ is Li-Yorke sensitive.

We emphasize that $\varepsilon$-scrambled is a stronger property than just scrambled. For example, [BHS, Proposition 5] shows the possibility of having scrambled sets that are not $\varepsilon$-scrambled for any $\varepsilon>0$.

Our concrete aim is to investigate the Lebesgue measure of the above sets for $C^{2}$ (or sometimes $C^{3}$ ) multimodal maps from the interval $I$ into itself with non-flat critical points (denoted by $C_{\mathrm{nf}}^{2}(I)$ and $C_{\mathrm{nf}}^{3}(I)$, respectively). The advantage of working in this setting is that there are many tools at hand to deal with measure-theoretic properties. Remarkably the most important of these tools is purely topological: maps from $C_{\mathrm{nf}}^{2}(I)$ have no wandering intervals, see [MS, Theorem A, p. 267].

As it happens, some of the results in the paper are based on a generalization of this property which is of interest in itself.

Definition 5. A point $x$ is asymptotically periodic, written $x \in$ AsPer, if there is a periodic point $p$ such that $\lim _{n \rightarrow \infty}\left|f^{n}(x)-f^{n}(p)\right|=0$. A measurable set $W$ is strongly wandering if $f^{n}(W) \cap f^{m}(W)=\emptyset$ for all $n>m \geq 0$ and $W$ contains no asymptotically periodic points. A wandering interval is just an interval which is strongly wandering.

The notion of strongly wandering set was introduced by Blokh and Lyubich in [BL] (in a slightly different way). Under the assumption of negative Schwarzian derivative, the non-existence of strongly wandering sets of positive measure was proved in the unimodal case (and stated in the multimodal case without inflection points) in [BL]. Here we prove it for maps from $C_{\mathrm{nf}}^{2}(I)$, for which inflection points are now allowed.

Theorem A. Let $f \in C_{\mathrm{nf}}^{2}(I)$. Then every strongly wandering set has zero Lebesgue measure.

Concerning the size of Li-Yorke chaos, the first natural question is whether smooth multimodal maps may have scrambled sets of positive Lebesgue measure. There is extensive 
literature on the subject. Examples of continuous maps possessing scrambled sets of positive or even full measure are well known: [K, $[\mathrm{S} 2, \mathrm{Mi},[\mathrm{BH}]$. In fact, if $f$ is chaotic (respectively, has a dense orbit), then it is topologically conjugate to a map having a positive (respectively, full) measure scrambled set [JK, S3] (respectively, [SS]).

Of course, it is not possible that the whole interval is scrambled (in fact, a scrambled set cannot be residual on any subinterval of $I$, see [G]), although there are maps with scrambled forward invariant Cantor sets [HY]. However such maps cannot be multimodal (see Proposition 26).

It is worth emphasizing that maps of type $2^{\infty}$ in the Sharkovskiy ordering (that is, those having periodic points of periods all powers of 2 , but no other periods) can possess scrambled sets of positive measure, but not of full measure because any Li-Yorke chaotic map of type $2^{\infty}$ has a wandering interval. Indeed, if a map of type $2^{\infty}$ has no wandering intervals, then all points are approximately periodic [S3]. However the following result is well known (see e.g. [BC, p. 144]):

Proposition 6. If $f: I \rightarrow I$ is continuous and $x, y$ are approximately periodic points, then $(x, y)$ is either asymptotic or distal.

Hence a scrambled set can contain at most one approximately periodic point. Finally, positive measure scrambled sets may also exist for $C^{\infty}$ maps (with flat critical points) or $C^{1}$ maps with non-flat critical points, but a $C^{1}$ map cannot have a full measure scrambled set [J1, J2, BJ2].

Nevertheless, it is a widely held view that these are rather pathological examples. For instance, it is known that neither maps from $C_{\mathrm{nf}}^{2}(I)$ with hyperbolic periodic points and whose critical points satisfy the Misiurewicz condition, nor maps in $C_{\mathrm{nf}}^{3}(I)$ with negative Schwarzian derivative and having no wild attractors, may possess scrambled sets of positive measure [BJ1, BrJ]. Our next result confirms these expectations:

Theorem B. If $f \in C_{\mathrm{nf}}^{3}(I)$, then it has no measurable scrambled sets of positive Lebesgue measure.

It seems rather paradoxical that scrambled sets have zero measure even in the case when there is an acip, but this is not really so. The key point is measurability. For instance, one can easily derive from [S1] that the full logistic map $f(x)=4 x(1-x)$ possesses a non-measurable scrambled set with full exterior Lebesgue measure. Since the sets Dis, Asymp, LY, $\mathrm{LY}_{\varepsilon}$, AsPer are all Borel, hence measurable sets [J3, BrJ], the moral is that we should measure these sets rather than scrambled sets. The idea of passing to the square $I \times I$ to study topological or measure-theoretic properties of Li-Yorke chaos is due to Lasota and was first used by Piórek $[\mathrm{P}]$.

To begin with, we prove that there almost no "non-trivial" asymptotic pairs.

Theorem C. If $f \in C_{\mathrm{nf}}^{2}(I)$, then Asymp $\backslash($ AsPer $\times$ AsPer) has zero (two-dimensional) Lebesgue measure.

We can consider Theorem $\mathrm{C}$ as a weak form of sensitivity to initial conditions in the absence of periodic attractors. It is really quite weak: it applies in particular to the 
infinitely renormalizable case where almost all points are attracted by a solenoidal set, so there is no sensitivity to initial conditions in the standard Guckenheimer sense [Gu]. What Theorem Cemphasizes is that there are no "privileged" routes (that is, with positive measure) to measure-theoretic attractors.

The Li-Yorke property describes how chaotically pairs of points behave with respect to each other, and is hence a property of the Cartesian product $\left(I^{2}, f_{2}\right)$, for $I^{2}=I \times I$ and $f_{2}(x, y):=(f \times f)(x, y)=(f(x), f(y))$. If $(x, y)$ is Li-Yorke, then orb $((x, y))$ accumulates on, but does not converge to, the diagonal of $I^{2}$. Hence LY is a weaker property than $(x, y)$ having a dense orbit in $I^{2}$. We want to find conditions ensuring that LY has positive (or full) mass w.r.t. two-dimensional Lebesgue measure $\lambda_{2}$. Recall the a map $g$ is called topologically mixing if every iterate $g^{n}$ has a dense orbit. According to Proposition 9, the orbit of Lebesgue a.e. point is either attracted by a periodic point or a solenoidal set, or eventually falls into an interval $K$ such that $f^{r}(K)=K$ for some $r$ and the restriction $g=\left.f^{r}\right|_{K}$ is topologically mixing. As we explained earlier, approximately periodic points cannot be used to produce Li-Yorke pairs. On the other hand, all sets from Definition 3 are the same for $f$ as for any of its iterates $f^{r}$. Thus we can restrict ourselves to the case where the map $f$ itself is topologically mixing.

Now, if $f$ is topologically mixing, then either almost all points have a dense orbit, or the orbits of almost all points are attracted by finitely many pairwise disjoint wild Cantor attractors. It turns out that in the first case $\lambda_{2}$-a.e. pair $(x, y)$ is Li-Yorke.

Theorem D. Let $f \in C_{\mathrm{nf}}^{3}(I)$ be a topologically mixing map having no Cantor attractors. Then the Cartesian product $\left(I^{2}, \lambda_{2}, f_{2}\right)$ is ergodic and for every $x \in I$ there is a full measure set $C_{x} \subset I$ such that

$$
\liminf _{n \rightarrow \infty}\left|f^{n}(y)-f^{n}(x)\right|=0, \quad \limsup _{n \rightarrow \infty}\left|f^{n}(y)-f^{n}(x)\right| \geq \operatorname{diam}(I) / 2,
$$

for every $y \in C_{x}$. In particular, $\mathrm{LY}_{1 / 2}$ has full measure and $f$ is Li-Yorke sensitive.

Weaker versions of this result under additional Misiurewicz or negative Schwarzian conditions were proved earlier [BJ1, BrJ]. Ergodicity of $\left(I^{2}, \lambda_{2}, f_{2}\right)$ is parallel to Lebesgue measure $\lambda$ being weak mixing, although in its standard definition, weak mixing applies to invariant measures only, see Subsection 2.4. If $f$ admits an acip, then we can say more: $\lambda_{2}$-a.e. pair $(x, y)$ has a dense orbit in $I^{2}$, see Corollary 28. However, it seems possible that there are cases where $f$ admits no acip, $\lambda_{2}$-a.e. pair $(x, y)$ is Li-Yorke, but has no dense orbit in $I^{2}$.

It remains to consider the case where $f \in C_{\mathrm{nf}}^{2}(I)$ is a topologically mixing map having a wild attractor $\mathcal{A}$. Let $\operatorname{Bas}(\mathcal{A})$ be the set of points whose orbit is attracted by $\mathcal{A}$. We already know (Theorem $(\mathrm{C})$ that Asymp has zero measure. Also, if some $x \in \operatorname{Bas}(\mathcal{A})$ is approximately periodic, then by Proposition $[31, \mathcal{A}$ is conjugate to an adding machine, so all points in $\operatorname{Bas}(\mathcal{A})$ are approximately periodic. Finally, recall that every pair of approximately periodic points is either asymptotic or distal. Our result in this area classifies which behaviors can (and indeed do) occur:

Theorem E. Let $f \in C_{\mathrm{nf}}^{2}(I)$ be a topologically mixing map with a wild attractor $\mathcal{A}$. Then one of the following alternatives must occur: 
(a) Lebesgue a.e. pair of points in $\operatorname{Bas}(\mathcal{A})$ is distal and every point in $\operatorname{Bas}(\mathcal{A})$ is approximately periodic;

(b) Lebesgue a.e. pair of points in $\operatorname{Bas}(\mathcal{A})$ is distal and no point in $\operatorname{Bas}(\mathcal{A})$ is approximately periodic;

(c) Lebesgue a.e. pair of points in $\operatorname{Bas}(\mathcal{A})$ is Li-Yorke;

(d) Both Dis and LY have positive Lebesgue measure in $\operatorname{Bas}(\mathcal{A}) \times \operatorname{Bas}(\mathcal{A})$.

There are examples of polynomial unimodal maps of all above types (a)-(d) so that additionally, in cases $(b)-(d), \operatorname{Bas}(\mathcal{A})$ contains $\varepsilon$-scrambled sets for a fixed $\varepsilon>0$ and $f$ is $L i$-Yorke sensitive on $\operatorname{Bas}(\mathcal{A})$.

\section{Preliminaries}

2.1. Interval maps. A continuous map $f: I \rightarrow I$ (for $I=[0,1]$ ) is called multimodal if $[0,1]$ can be decomposed into finitely many subintervals on which $f$ is (strictly) monotone. A point $c$ is critical if $f^{\prime}(c)=0$; the set of critical points is denoted by Crit. Critical points can be turning points (if $f$ assume a local extremum at $c$ ) or inflection points, and hence there can be more critical points than maximal intervals of monotonicity. A differentiable map having exactly one critical (turning) point is called unimodal. Near a turning point $c$, there is a largest interval $[a, b]$ such that $f(a)=f(b)$ and $f$ is monotone on each of the intervals $[a, c]$ and $[c, b]$. Then there is a continuous involution $\tau_{c}:[a, b] \rightarrow[a, b]$ such that $f\left(\tau_{c}(x)\right)=f(x)$ and $\tau_{c}(x) \neq x$ for every $x \neq c$.

We say that $f \in C_{n f}^{k}(I)$ if $f: I \rightarrow I$ has a finite critical set Crit, is $C^{k}$ and each critical point is non-flat, i.e., for each $c \in$ Crit, there exist a $C^{k}$ diffeomorphism $\varphi_{c}$ with $\varphi_{c}(c)=0$ and an $\ell_{c} \in(1, \infty)$, called the critical order of $c$, such that $f(x)= \pm\left|\varphi_{c}(x)\right|^{\ell_{c}}+f(c)$ for $x$ close to $c$. It follows that if $n$ is the smallest integer $\geq \ell_{c}$, then $D^{m} f(c)=0$ for $1 \leq m<n$, but $D^{n} f(c) \neq 0$. Conversely, if $f$ is $C^{k+1}$ near $c$ and $D^{n} f(c) \neq 0$ for some $2 \leq n \leq k+1$, then $c$ is non-flat.

We can always enlarge the domain of $f$ (without adding new critical points) and rescale such that

$$
f(\partial I) \subset \partial I \quad \text { and } \quad \text { Crit } \cap \partial I=\emptyset .
$$

Observe that this operation does not change the zero or positive measure quality of the sets from Definition [3. Hence, except when $f$ is topologically mixing, we will always assume that (11) holds. Also, we will assume without loss of generality that

Crit does not contain any periodic point,

because if $c$ is a periodic critical point, then we can modify slightly $f$ near the orbit of $c$ so that the resulting map has a periodic orbit containing no critical points and attracting the same points as previously attracted by the orbit of $c$.

Recall that the map $f$ is topologically mixing if every iterate $f^{n}$ has a dense orbit. This excludes the case that there is a proper compact subinterval $J$ of $I$ such that $f^{r}(J) \subset J$ for some $r \geq 1$ (i.e., $f$ is non-renormalizable). The map $f$ is topologically exact (also called locally eventually onto) if for every non-degenerate interval $J \subset I$, there is $n$ such that $f^{n}(J)=I$. For multimodal maps, topologically mixing and topologically exact are equivalent, see e.g. [BC, pp. 157-158]. 
2.2. Attractors of interval maps. The orbit $\left\{f^{n}(x)\right\}_{n=0}^{\infty}$ of a point $x$ is denoted by $\operatorname{orb}(x)$. More generally, the orbit of a set $A$ is $\operatorname{orb}(A):=\bigcup_{n=0}^{\infty} f^{n}(A)$. The $\omega$-limit set $\omega(x):=\bigcap_{n \in \mathbb{N}} \mathrm{Cl}_{m \geq n} f^{n}(x)$ of $x$ is the set of limit points of the orbit of $x$. If $\mathcal{A}$ is a subset of $I$, then we call $\operatorname{Bas}(\mathcal{A})=\{x \in I: \omega(x) \subset \mathcal{A}\}$ the basin (of attraction) of $\mathcal{A}$. A periodic orbit $\mathcal{O}$ is called attracting if its basin contains an open set. The union of the components of $\operatorname{Bas}(\mathcal{O})$ intersecting $\mathcal{O}$ is called the immediate basin of $\mathcal{O}$. If $\operatorname{Bas}(\mathcal{O})$ contains a neighborhood of $\mathcal{O}$, then $\mathcal{O}$ is called a two-sided attracting periodic orbit; otherwise it is called a one-sided attracting periodic orbit. If $p$ is a periodic point of period $r$ and $\left|D f^{r}(p)\right|$ is less than, equal to, or greater than 1 , then the orbit of $p$ is called hyperbolic attracting, parabolic, or hyperbolic repelling respectively. Of course, only hyperbolic attracting and parabolic orbits can be attracting. If $f \in C_{\mathrm{nf}}^{2}(I)$, then it is well known that all periodic orbits of sufficiently high period are hyperbolic repelling [MMS]. Furthermore, maps in $C_{\mathrm{nf}}^{2}(I)$ have no wandering intervals. One of the consequences of this is the following useful result. Below $\operatorname{dist}(A, B)$ denotes the distance between the sets $A$ and $B$ (by convention $\operatorname{dist}(A, \emptyset)=\infty$ ).

Proposition 7 (The non-Contraction Principle). Let $f: I \rightarrow I$ be a multimodal map without wandering intervals. Then for every $\varepsilon>0$ there is $\delta>0$ such that if $J$ is an interval such that $|J|<\delta$ and $\operatorname{dist}(J, p)>\varepsilon$ for each attracting periodic point $p$, then every component of every preimage $f^{-n}(J)$ of $J$ has length less than $\varepsilon$.

Proof. This follows easily from the Contraction Principle as stated in [MS, p. 305]. The principle is a bit of a misnomer, so we added non- in our version.

Definition 8. We call a closed invariant set $\mathcal{A}$ a (measure-theoretic) attractor if its basin has positive Lebesgue measure, and there is no proper subset $\mathcal{A}^{\prime} \subset \mathcal{A}$ with the same properties.

Note that $\mathcal{A}$ need not be an attractor in any topological sense: the $\operatorname{basin} \operatorname{Bas}(\mathcal{A})$ need not contain a neighborhood of $\mathcal{A}$, nor be of second Baire category.

In order to understand the nature of measure-theoretic attractors of maps from $C_{\mathrm{nf}}^{2}(I)$, certain types of interval cycles are of particular interest. We say that a compact interval $K$ is periodic (of period $r$ ) if $K, \ldots, f^{r-1}(K)$ have disjoint interiors and $f^{r}(K) \subset K$. We call the union $\bigcup_{i=0}^{r-1} f^{i}(K)$ a cycle of intervals and denote it by $\operatorname{cyc}(K)$. If $K_{0} \supset K_{1} \supset \cdots$ is a nested sequence of periodic intervals of periods $r_{0}<r_{1}<\cdots$, then $S=\bigcap_{i=0}^{\infty} \operatorname{cyc}\left(K_{i}\right)$ is called a solenoidal set and all points from $S$ are called solenoidal points. If $r_{i+1}=2 r_{i}$ for every $i$ sufficiently large, then we say that both $S$ and its points are of Feigenbaum type. If $i_{0}$ is such that $\operatorname{cyc}\left(K_{i_{0}}\right) \cap$ Crit $=S \cap$ Crit and all periodic points in $\operatorname{cyc}\left(K_{i_{0}}\right)$ are hyperbolic repelling, then we say that $\operatorname{cyc}\left(K_{i_{0}}\right)$ is solenoidal. Thus all solenoidal sets are of Cantor type and all cycles $\operatorname{cyc}\left(K_{i}\right)$ are solenoidal if $i$ is sufficiently large.

A compact invariant set $A$ is Lyapunov stable if for every neighborhood $U$ of $A$, there exists a neighborhood $V$ of $A$ such that $f^{n}(V) \subset U$ for every $n$. We have the following classification of measure-theoretic attractors, see [M], Ke, BL, Ly1, MS, SV].

Proposition 9. If $f \in C_{\mathrm{nf}}^{2}(I)$, then $f$ has countably many attractors $\mathcal{A}$, which are of the following types:

(1) $\mathcal{A}$ is an attracting periodic orbit; 
(2) $\mathcal{A}$ is a cycle of intervals on which Lebesgue a.e. orbit is dense;

(3) $\mathcal{A}$ is a solenoidal set (the infinitely renormalizable case). Then $\mathcal{A}$ is Lyapunov stable and the basin $\operatorname{Bas}(\mathcal{A})$ is of second Baire category.

(4) $\mathcal{A}$ is a minimal Cantor set, but not of the above type. In particular, $\mathcal{A}$ is not Lyapunov stable, and $\operatorname{Bas}(\mathcal{A})$ is of first Baire category.

For Lebesgue a.e. $x \in I$, either $x$ has a finite orbit (that is, $x$ is eventually periodic) or $\omega(x)$ is one of the attractors above, and the number of attractors of type (2)-(4) together is no more than the number of critical points (because each of them must contain at least one critical point).

More can be said: there can be countably many disjoint cycles, but all but finitely many of them must be disjoint from the basins of periodic attractors. If a cycle is solenoidal, then almost all its orbits are attracted by the solenoidal set contained in the cycle. If a cycle $\operatorname{cyc}(K)$ contains a dense orbit, then one of the following two holds:

- The whole cycle is an attractor and almost all its points are dense in $\operatorname{cyc}(K)$. If $K$ has period $r$ this can still mean that $K$ consists of two intervals $J$ and $J^{\prime}$ with a common boundary point such that $f^{r}(J)=J^{\prime}$ and $f^{r}\left(J^{\prime}\right)=J$. In this case $f^{2 r}$ is topologically mixing on $J$. Otherwise $f^{r}$ is topologically mixing on $K$.

- The cycle contains finitely many attractors of type (4) attracting the orbits of almost all points in the cycle (hence almost no point has a dense orbit in the cycle).

An attractor of type (3) or (4) is called a solenoidal and a wild attractor respectively. Proposition 9 implies that the orbit of $\lambda$-a.e. $x \notin$ AsPer accumulates on Crit, so every solenoidal set is in fact a solenoidal attractor. It is well known that attractors of type (1) and (3) are uniquely ergodic, and it follows from [BSS, Theorem 4] that this is also true for attractors of type (4). The existence of wild attractors has been proved for unimodal maps only if the combinatorial properties of the map are very specific, and the critical order $\ell_{c}$ is sufficiently large $\left(\ell_{c} \gg 2\right)$. The prototype is the unimodal Fibonacci map [BKNS], but there are other Fibonacci-like combinatorics that allow wild attractors, see [Br3]. For multimodal maps, there are combinatorial types that allow wild attractors also if all critical orders are $\ell_{c}=2[\mathrm{vS}]$.

2.3. Distortion results. In what follows we denote by $|A|$ or $\lambda(A)$ the Lebesgue measure of a measurable set $A \subset I$ (also, $\lambda_{2}$ will denote the two-dimensional Lebesgue measure). The density of a set $X$ in $J$ is $|X \cap J| /|J|$. A point $x$ is a (Lebesgue) density point of $X$ if $\lim _{\varepsilon \rightarrow 0}|X \cap(x-\varepsilon, x+\varepsilon)| / 2 \varepsilon=1$.

Many of the arguments in this paper rely on measuring images under $f^{n}$ of neighborhoods $U$ of density points of certain sets. If $\left.f^{n}\right|_{U}$ is diffeomorphic then the Koebe Principle (see Proposition 13) is used to estimate how densities change, but in general, $U$ can visit several critical points in its first $n$ iterates. In this case, we need more advanced techniques and results (relying on work in [BM1, MS, SV]), which we summarize below in Theorems 20 and 21 , 
We call a sequence $\left(G_{i}\right)_{i=0}^{l}$ of intervals a chain if $G_{i}$ is a maximal interval such that $f\left(G_{i}\right) \subset G_{i+1}, i=0, \ldots, l-1$. If $\left(H_{i}\right)_{i=0}^{l}$ and $\left(G_{i}\right)_{i=0}^{l}$ are chains and $H_{i} \subset G_{i}$ for every $i$, then we will write $\left(H_{i}\right)_{i=0}^{l} \subset\left(G_{i}\right)_{i=0}^{l}$. If $x \in G_{0}$ (or $J$ is a subinterval of $G_{0}$ ), then we call $\left(G_{i}\right)_{i=0}^{l}$, or sometimes just the interval $G_{0}$, the pullback (chain) of $G_{n}$ along $x, \ldots, f^{l}(x)$ (or along $\left.J, \ldots, f^{l}(J)\right)$. The order of a chain is the number of intervals $G_{i}, 0 \leq i<l$, intersecting Crit.

Remark 10. Under the hypothesis $f \in C_{\mathrm{nf}}^{2}(I)$, if $G_{l}$ is a small interval not too close to any attracting periodic orbit, then all intervals $G_{i}, i<l$, are also very small by Proposition 7 . (Notice that the closure of the set of attracting periodic points only contains periodic points because the periods of attracting orbits are bounded and recall that Crit only contain nonperiodic points, see assumption (2).)

Given intervals $J \subset K$, we say that $J$ is $\xi$-well inside $K$ if the components $L$ and $R$ of $K \backslash J$ satisfy $|L|,|R| \geq \xi|J|$. If in addition $\xi|L| \leq|R|$ and $\xi|R| \leq|L|$, then we say that $J$ is $\xi$-well centered in $K$.

A differentiable map without critical points $f: J \rightarrow \mathbb{R}$ has distortion bounded by $\kappa>0$ if

$$
\sup _{x, y \in J} \frac{\left|f^{\prime}(x)\right|}{\left|f^{\prime}(y)\right|} \leq \kappa
$$

We emphasize that if the density of a subset $X$ of $J$ is very close to 1 , say $>1-\varepsilon$, then the density of $f(X)$ in $f(J)$ is $>1-\kappa \varepsilon$, so it is very close to 1 as well.

An open subset $V$ of $\mathbb{R}$ is called nice if $\operatorname{orb}(\partial V) \cap V=\emptyset$. The first entry map to a nice set $V$ is the map $\phi_{V}: D(V) \rightarrow V$ defined on the domain $D(V)=\cup_{n \geq 1} f^{-n}(V)$ by $\phi_{V}(x)=f^{r_{V}}(x)$, where $r_{V}=\min \left\{k>0: f^{k}(x) \in V\right\}$ is the first entry time. The maximal intervals $J$ on which the first entry time $r_{V}$ is constant are called entry domains. By convention we assume that $J$ does not intersect $V$ : if $J$ is a subset of a component of $V$ with first entry time $r_{V}(J) \equiv r$, then we prefer to call $J$ a return domain with return time $r$. The main reason why nice sets are "nice" is that the return and entry domains are all disjoint. Furthermore, all components in the backward orbit of a nice set are nice, and two such intervals are either nested or disjoint.

Lemma 11. Let $f \in C_{\mathrm{nf}}^{2}(I)$. Then for every $\xi>0$ there exists $\xi^{\prime}=\xi^{\prime}(\xi, f)>0$ such that if $T$ is a component of the preimage of an interval $V$ and $U$ is an interval $\xi$-well inside $V$ (respectively, $\xi$-well centered in $V$ ), then the preimage $J$ of $U$ in $T$ is $\xi^{\prime}$-well inside $T$ (respectively, $\xi^{\prime}$-well centered in $T$ ).

Proof. This follows easily from [BM1, Lemmas 3.2 and 3.3].

The Schwarzian derivative of a $C^{3}$ map $f$ is defined for every $x \notin$ Crit by

$$
S f(x)=\frac{f^{\prime \prime \prime}(x)}{f^{\prime}(x)}-\frac{3}{2}\left(\frac{f^{\prime \prime}(x)}{f^{\prime}(x)}\right)^{2} .
$$

A $C^{1}$ version reads: $f$ has negative Schwarzian derivative if $1 / \sqrt{\left|f^{\prime}\right|}$ is convex on every interval where it is defined. If $f$ has negative Schwarzian derivative, then we can frequently estimate distortion using the Koebe Principle, see [MS, Section IV.1] or [BM1, "Koebe lemma"]: 
Proposition 12 (Koebe Principle for negative Schwarzian derivative maps). If $f: G \rightarrow$ $f(G)$ is a diffeomorphism with negative Schwarzian derivative, $H \subset G$ and $f(H)$ is $\xi$-well inside $f(G)$, then $H$ is $\xi^{3} /\left(2(3 \xi+2)^{2}\right)$-well inside $G$ and $\left.f\right|_{G}$ has distortion bounded by $((1+\xi) / \xi)^{2}$.

However, in this paper we will use also use a $C^{2}$-version of these classical results.

Proposition 13 ( $C^{2}$ Koebe Principle). Given $f \in C_{\mathrm{nf}}^{2}(I)$, there is a function $Q$ : $(0, \infty) \rightarrow(0, \infty)$ with $\lim _{\varepsilon \rightarrow 0} Q(\varepsilon)=0$ such that the following holds. Suppose that $H \subset G$ are intervals such that $\left.f^{l}\right|_{G}$ is a diffeomorphism and $f^{l}(H)$ is $\xi$-well-inside $f^{l}(G)$ for some $\xi>0$. Then there exists $\xi^{\prime}=\xi^{\prime}(\xi, f)>0$ (with $\xi^{\prime} \rightarrow \infty$ as $\left.\xi \rightarrow \infty\right)$, and

$$
\kappa=\exp \left(Q\left(\max _{0 \leq i<l}\left|f^{i}(G)\right|\right) \cdot \sum_{i=0}^{l-1}\left|f^{i}(H)\right|\right) \cdot\left(\frac{1+\xi}{\xi}\right)^{2}
$$

such that the distortion of $\left.f^{l}\right|_{H}$ is bounded by $\kappa$ and $H$ is $\xi^{\prime}$-well inside $G$.

Proof. See [SV, Proposition 2].

We have an obvious bound $\sum_{i=0}^{l-1}\left|f^{i}(H)\right| \leq 1$ when the $f^{i}(H), 0 \leq i<l$, are pairwise disjoint. This is what we will use in the following corollary, which uses Lemma 11 and Proposition 13 as well.

Corollary 14. Let $f \in C_{\mathrm{nf}}^{2}(I)$. Then for any $\xi>0$ and $k \geq 0$, there are $\xi^{\prime}=\xi^{\prime}(\xi, k, f)>$ $0, \sigma=\sigma(\xi, k, f)>0$ such that the following statement holds: Let $\left(H_{i}\right)_{i=0}^{l} \subset\left(G_{i}\right)_{i=0}^{l}$ be chains such that $\left(G_{i}\right)$ has order at most $k, G_{l}$ is a small interval close enough to Crit, and the intervals $H_{i}$ are pairwise disjoint. If $H_{l}$ is $\xi$-well inside $G_{l}$, then $H_{0}$ is $\xi^{\prime}$-well inside $G_{0}$. If in addition $k=0$, then there is $\kappa=\kappa(\xi, f)>0$ such that $\left.f^{l}\right|_{H_{0}}$ has distortion bounded by $\kappa$.

Proof. By saying that " $G_{l}$ is a small interval close enough Crit" we mean that there is $\varepsilon_{0}=\varepsilon_{0}(f)$ such that $\left|G_{l}\right|<\varepsilon_{0}$ and $\operatorname{dist}\left(G_{l}, c\right)<\varepsilon_{0}$ for some non-periodic critical point $c$, where $\varepsilon_{0}$ is chosen so that, if $\varepsilon_{1}$ satisfies $Q(\varepsilon)<1$ for every $\varepsilon \leq \varepsilon_{1}$ in Proposition [13, then $\left|G_{i}\right|<\varepsilon_{1}$ for every $i<l$ (see Remark 10).

The case $k=0$ is just Proposition 13. Let us give the proof for $k=1$; the idea is the same for $k>1$. Let $G_{t}$ be the interval from the chain containing the critical point. Now we construct three subchains, $\left(H_{i}\right)_{i=0}^{t} \subset\left(G_{i}\right)_{i=0}^{t},\left(H_{i}\right)_{i=t}^{t+1} \subset\left(G_{i}\right)_{i=t}^{t+1}$ and $\left(H_{i}\right)_{i=t+1}^{l} \subset\left(G_{i}\right)_{i=t+1}^{l}$. Applying Proposition 13 to the third chain, we find $\xi_{1}=\xi_{1}(\xi, f)$ and $\kappa_{1}=\kappa_{1}(\xi, f)$ such that $H_{t+1}$ is $\xi_{1}$-well inside $G_{t+1}$. Applying Lemma 11 to the middle chain, we find $\xi_{2}=\xi_{2}\left(\xi_{1}, f\right)$ such that $H_{t}$ is $\xi_{2}$-well inside $G_{t}$. Finally, applying again Proposition 13 to the first chain, we find $\xi=\xi\left(\xi_{2}, f\right)$ such that $H_{0}$ is $\xi$-well inside $G_{0}$.

The Koebe property refers to distortion control in the presence of Koebe space. Slightly weaker is the Macroscopic Koebe property, which refers to the preservation of Koebe space under pullback. Hence the fact that $H$ is $\xi^{\prime}$-well inside $G$ in Proposition 13 is basically a Macroscopic Koebe statement. 
In order to use the above results, we need conditions guaranteeing the existence of Koebe space at the end of chains. The following propositions are particularly useful in this regard.

Proposition 15. Let $f \in C_{\mathrm{nf}}^{2}(I)$. Then for every $\xi>0$ there exists $\xi^{\prime}=\xi^{\prime}(\xi, f)>0$ such that if $V$ and $U$ are nice intervals, $U$ is $\xi$-well inside $V, x \in V$ and $f^{k}(x) \in U$ (with $k \geq 1$ not necessarily minimal), then the pullback of $U$ along $x, \ldots, f^{k}(x)$ is $\xi^{\prime}$-well inside the return domain to $V$ containing $x$.

In particular, if $U$ is a return domain to $V$ which is $\xi$-well inside $V$, then all return domains to $U$ are $\xi^{\prime}$-well inside $U$.

Proof. This is Theorem C(1) from [SV] (see also the remark below Theorem C(1) and the erratum to that paper). The second statement follows easily from the first one, by fixing an arbitrary return domain $K$ to $U$ and $x \in K$, and choosing $k$ as the return time of $K$. The interval $K$ is then the pullback of $U$ along $x, \ldots, f^{k}(x)$ and $U$ is the return domain to $V$ containing $x$.

Proposition 16. Let $f \in C_{\mathrm{nf}}^{2}(I)$ and let $x$ be a recurrent point of $f$ which is neither periodic nor of Feigenbaum type. Then there are $\xi_{0}=\xi_{0}(f)>0$ and an arbitrarily small nice neighborhood $J$ of $x$ such that the return domain to $J$ containing $x$ is $\xi_{0}$-well inside $J$. Assume in addition that $x$ is not solenoidal, and that $I_{0}$ is a nice neighborhood of $x$ so small that it contains no periodic neighborhood of $x$. Let $\left(I_{m}\right)_{m=0}^{\infty}$ be the sequence of nice intervals such that $I_{m}$ the return domain to $I_{m-1}$ containing $x$. In this case, there are infinitely many $m$ such that $I_{m+1}$ is $\xi_{0}$-well inside $I_{m}$.

Proof. This is a mixture of Theorems A(1) and A'(2) from [SV].

2.4. Notions from ergodic theory. Let $X$ be a topological space with Borel $\sigma$-algebra $\mathcal{B}$ and let $f: X \rightarrow X$ be a Borel measurable map. Recall that a probability measure $\mu$ on $\mathcal{B}$ is called invariant (respectively, non-singular) if $\mu\left(f^{-1}(A)\right)=\mu(A)$ (respectively, $\mu(A)=0$ if and only if $\left.\mu\left(f^{-1}(A)\right)=0\right)$ for any $A \in \mathcal{B}$. In what follows we assume that $\mu$ is non-singular but not necessarily invariant.

Definition 17. Let $(X, \mu, f)$ be defined as above. Write $X^{2}:=X \times X, \mu_{2}:=\mu \times \mu$, $f_{2}:=f \times f$ and let $\mathcal{B}_{2}$ be the Borel $\sigma$-algebra in $X \times X$. We call the system $(X, \mu, f)$

- conservative if for every set $A \in \mathcal{B}$ with $\mu(A)>0$, there is $n \geq 0$ such that $\mu\left(A \cap f^{n}(A)\right)>0$;

- ergodic if $f^{-1}(A)=A \in \mathcal{B}$ implies $\mu(A)=0$ or 1 . If $f$ is ergodic and conservative, then $\mu$-a.e. orbit is dense in $\operatorname{supp}(\mu)$.

- exact if $f^{-n}\left(f^{n}(A)\right)=A \in \mathcal{B}$ for every $n \geq 0$ implies $\mu(A)=0$ or 1 ;

- mixing if $\mu$ is invariant and

$$
\lim _{n \rightarrow \infty} \mu\left(A \cap f^{-n}(B)\right)=\mu(A) \mu(B)
$$

for all sets $A, B \in \mathcal{B}$;

- weak mixing if $\mu$ is invariant, but 1 is the only eigenvalue corresponding to a measurable eigenfunction of the operator $P: L^{1}(\mu) \rightarrow L^{1}(\mu)$ defined by $P \psi=\psi$ o $f^{-1}$. Weak mixing invariant measures have, in fact, several equivalent definitions. 
One of them is that the Cartesian product $(X \times Y, \mu \times \nu, f \times g)$ is ergodic for every ergodic measure preserving system $(Y, g, \nu)$. (In particular, weak mixing implies that $\left(X^{2}, \mu_{2}, f_{2}\right)$ is ergodic.)

- For non-invariant measures, we can still speak of mildly mixing: A non-singular probability measure is mildly mixing if for every set $A \in \mathcal{B}$ of positive measure

$$
\liminf _{n \rightarrow \infty} \mu\left(A \cap f^{-n}(A)\right)>0 .
$$

Mild mixing implies that $\left(X^{2}, \mu_{2}, f_{2}\right)$ is ergodic. (If $f$ is invertible, then mild mixing is equivalent to $\left(X^{2}, \mu_{2}, f_{2}\right)$ being ergodic. In this case $f$ also preserves a probability measure equivalent to $\mu$, but neither of these stronger statements holds in general if $f$ is non-invertible. See [HS] for more results.)

Lemma 18. If $(X, \mu, f)$ is exact, then $\left(X^{2}, \mu_{2}, f_{2}\right)$ is ergodic.

Proof. Assume by contradiction that $\left(X^{2}, \mu_{2}, f_{2}\right)$ is not ergodic, so there is $U \in \mathcal{B}_{2}$ such that $f_{2}^{-1}(U)=U$ and $0<\mu_{2}(U)<1$. Then there is $a \in X$ such that $0<\mu\left(U_{a}\right)<1$ for $U_{a}=\{y \in X:(a, y) \in U\}$. Let $V_{a}=X \backslash U_{a}$. Then $f^{n}\left(U_{a}\right) \cap f^{n}\left(V_{a}\right)=\emptyset$ for all $n \geq 0$, so $\left(f^{-n} \circ f^{n}\right)\left(U_{a}\right)=U_{a}$ for all $n$, contradicting that $\mu$ is exact.

Lemma 19. If $X$ is separable and $(X, \mu, f)$ is ergodic and conservative, then $\mu$-a.e. $x$ has a dense orbit in $X$.

Proof. Let $\left\{U_{n}\right\}_{n \in \mathbb{N}} \subset \mathcal{B}$ be a countable basis of $X$, and set $Y_{n}:=\left\{x \in X: f^{k}(x) \in\right.$ $U_{n}$ infinitely often $\}$. Then $f^{-1}\left(Y_{n}\right)=Y_{n}$, so $\mu\left(Y_{n}\right)=0$ or 1 for each $n$. If $\mu\left(Y_{n}\right)=1$ for each $n$, then $Y:=\bigcap_{n} Y_{n}$ has full measure, and every $x \in Y$ has a dense orbit.

So assume that $n$ is such that $\mu\left(Y_{n}\right)=0$. For $Z=X \backslash Y_{n}$, we can write $Z=\bigcup_{k \in \mathbb{N}} Z_{k}$ where $Z_{k}=\left\{x \in X: k=\max \left\{i: f^{i}(z) \in U_{n}\right\}\right\}$. Since $\mu(Z)=1$, there is $k$ such that $\mu\left(Z_{k}\right)>0$, and hence $\mu\left(f^{k}\left(Z_{k}\right)\right)>0$. But then $f^{k}\left(Z_{k}\right) \subset U_{n}$ is a set of positive measure that never visits $U_{n}$ again, and this contradicts that $\mu$ is conservative.

The following diagram summarizes the implications between these various notions of mixing. The notions on the bottom line can be defined for non-invariant measures $\mu$, but any implication to the top line requires that $\mu$ is $f$-invariant and in particular conservative.

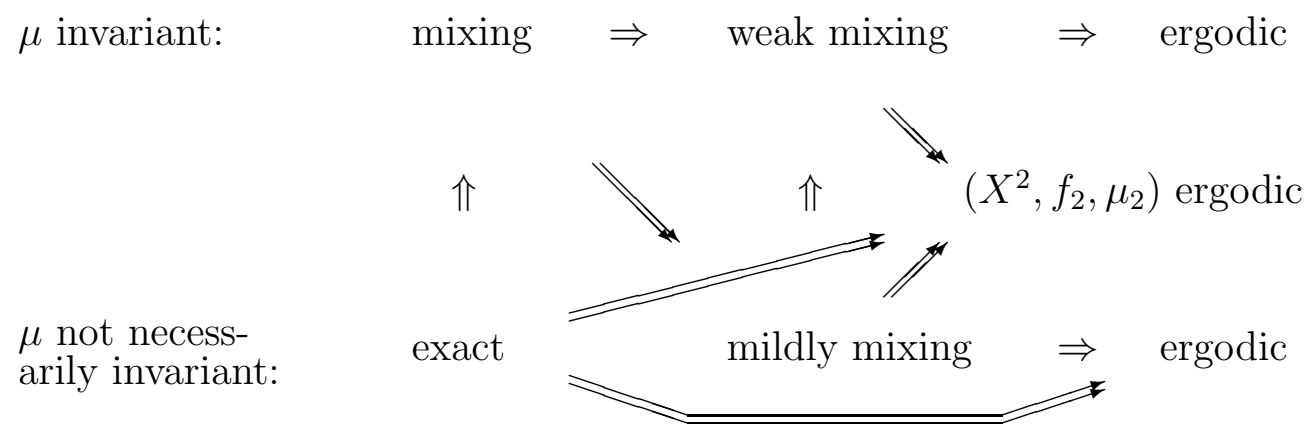




\section{INDUCING TO CRITICAL NEIGHBORHOODS AND STRONGLY WANDERING SETS}

In this section, we prove that strongly wandering sets have zero measure as a consequence of Theorem 20 below. Theorem 20 is a refinement of Theorem 1 of [CL] (where the slightly stronger hypothesis $f \in C_{\mathrm{nf}}^{3}(I)$ is used), which in turns improves Theorem D of [SV].

Theorem 20. Let $f \in C_{\mathrm{nf}}^{2}(I)$. Then there are positive constants $\xi=\xi(f), \kappa=\kappa(f)$, $\delta=\delta(f)$ and, for every $\varepsilon>0$ and $c \in$ Crit, open intervals $c \in U_{c} \subset V_{c}$, with $\left|V_{c}\right|<\varepsilon$, such that the following conditions hold:

(i) The set $U=\bigcup_{c \in \text { Crit }} U_{c}$ is nice and $U_{c}$ is $\xi$-well inside $V_{c}$ for every $c \in$ Crit.

(ii) If $J$ is an entry domain of the first entry map $\phi$ to $U$, say $\left.\phi\right|_{J}=\left.f^{j}\right|_{J}$ and $\phi(J)=$ $U_{c}$, then there is $K \supset J$ such that $\left.f^{j}\right|_{K}$ is a diffeomorphism and $f^{j}(K)=V_{c}$. Moreover, $\left.\phi\right|_{J}$ has distortion bounded by $\kappa$.

(iii) If $c \in$ Crit, then there are an open interval $W_{c} \subset U_{c}$ and $k_{c} \in \mathbb{N}$ such that $\left|W_{c}\right|>\delta\left|U_{c}\right|,\left.f^{k_{c}}\right|_{W_{c}}$ is a diffeomorphism with distortion bounded by $\kappa$, and

- either $W_{c} \subset f^{k_{c}}\left(W_{c}\right) \subset U_{c}$ (if $c$ is of Feigenbaum type);

- or $k_{c}=1$ and $\partial W_{c} \cap D(U)=\emptyset$ (if $c$ is not of Feigenbaum type).

The existence of the sets $U_{c} \subset V_{c}$ from Theorem 20 suggests the construction of an induced Markov map $F: U \rightarrow U$ with all branches mapping monotonically onto a component of $U$. The first, very detailed, constructions of such induced maps go back to Jakobson Ja], but the abstract statement for unimodal maps having no periodic or Cantor attractors comes from Martens' PhD. thesis [Ma]. During the writing of this paper, we learnt about a multimodal $C^{3}$ version by $\mathrm{Cai}$ and $\mathrm{Li}$ [CL, see Proposition 45, Their construction precludes the existence of parabolic points, hence we improve it slightly by showing the version below, where Crit $^{\prime}$ denotes the set of critical points interior to metric attractors of type (2) in Proposition 9 .

Let $E:=\bigcup_{n \geq 0} f^{-n}(A)$ where $A$ is the union of all attractors of type (2) in Proposition 9, that is, the union of cycles of intervals in which $\lambda$-a.e. orbit is dense.

Theorem 21. Let $f \in C_{\mathrm{nf}}^{3}(I)$ and let $U_{c}, V_{c}, c \in \mathrm{Crit}^{\prime}$, be defined as in Theorem 20 for $\varepsilon$ sufficiently small and let $U^{\prime}=\bigcup_{c \in \mathrm{Crit}^{\prime}} U_{c}$. Then for Lebesgue a.e. $x \in E$ we can find $k_{x} \in \mathbb{N}$ and intervals $G_{x} \supset H_{x} \ni x$ (with either $H_{x}=H_{y}$ or $H_{x} \cap H_{y}=\emptyset$ ) such that $f^{k_{x}}: G_{x} \rightarrow V_{c}$ is diffeomorphic for some $c \in \mathrm{Crit}^{\prime}$ and $f^{k_{x}}\left(H_{x}\right)=U_{c}$.

Let $F: U^{\prime} \rightarrow U^{\prime}$ be defined by $\left.F\right|_{H_{x}}=f^{k_{x}}: H_{x} \rightarrow U_{c}$ for the appropriate $c \in$ Crit' $^{\prime}$. Then all iterates of $F$ is well defined $\lambda$-a.e and its branches have uniformly bounded distortion, i.e., there is $\kappa=\kappa(f)>0$ such that for every $n \in \mathbb{N}$ and every interval $H$ on $\left.F^{n}\right|_{H}: H \subset U \rightarrow U_{c}$ is a diffeomorphism, the distortion of $\left.F^{n}\right|_{H}$ is bounded by $\kappa$.

We postpone the somewhat technical proofs of Theorems 20 and 21 to the appendix.

Proof of Theorem $\underline{A}$. Let $f \in C_{\mathrm{nf}}^{2}(I)$ and assume that $W$ is a strongly wandering set of positive measure. Let $x$ be a density point of $W$. It is not restrictive to assume that $x$ belongs to the interior of a cycle cyc $(K)$ which either contains a dense orbit or is of solenoidal type. We can also assume that orb $(x)$ accumulates on Crit. 
Let $\tau>0$ be such that $(x-\tau, x+\tau) \subset \operatorname{cyc}(K)$. We can assume that the density of $W$ in any subinterval of $(x-\tau, x+\tau)$ containing $x$ is larger than $1-\varepsilon_{0}$, with $\varepsilon_{0}>0$ so small that if $d=$ \# Crit, then $1-\delta^{-d} \kappa^{2 d+1} \varepsilon_{0}>1 / 2$, where $\delta=\delta(f)$ and $\kappa=\kappa(f)$ are the numbers from Theorem 20. Finally we fix $\varepsilon>0$ such that if $U$ is defined as in Theorem 20, then all components of $U$ and entry domains to $U$ have length less than $\tau$ (by the non-Contraction Principle).

Let $J_{1}$ be either the entry domain to $U$ containing $x$, say $\left.\phi\right|_{J_{1}}=\left.f^{j_{1}}\right|_{J_{1}}, f^{j_{1}}\left(J_{1}\right)=U_{c_{1}}$, or the component $U_{c_{1}}$ of $U$ containing $x$ (then we take $\left.j_{1}=0\right)$. Since $J_{1} \subset(x-\tau, x+\tau) \subset \operatorname{cyc}(K)$ and $\operatorname{cyc}(K)$ is invariant, the density of $W$ in $J_{1}$ is $>1-\varepsilon_{0}$ and $U_{c_{1}} \subset \operatorname{cyc}(K)$.

Let $W_{c_{1}}$ be the interval from Theorem 20, part (iii). Since $\left|W_{c_{1}}\right|>\delta\left|U_{c_{1}}\right|$ and the density of $f^{j_{1}}(W)$ in $U_{c_{1}}$ is $>1-\kappa \varepsilon_{0}$, the density of $f^{j_{1}}(W)$ in $W_{c_{1}}$ is $>1-\delta^{-1} \kappa \varepsilon_{0}$ and the density of $f^{j_{1}+k_{1}}(W)$ in $f^{k_{1}}\left(W_{c_{1}}\right)$ is $>1-\delta^{-1} \kappa^{2} \varepsilon_{0}$, where $k_{1}=k_{c_{1}}$ is as in Theorem 20(iii). If $c_{1}$ is a Feigenbaum critical point, then $W_{c_{1}} \subset f^{k_{c_{1}}}\left(W_{c_{1}}\right) \subset U_{c_{1}}$, so the density of $f^{j_{1}}(W)$ in $f^{k_{c_{1}}}\left(W_{c_{1}}\right)$ is also $>1-\delta^{-1} \kappa \varepsilon_{0}$. Therefore the densities of both $f^{j_{1}}(W)$ and $f^{j_{1}+k_{1}}(W)$ in $f^{k_{c_{1}}}\left(W_{c_{1}}\right)$ are $>1 / 2$, hence $f^{j_{1}}(W) \cap f^{j_{1}+k_{1}}(W) \neq \emptyset$, contradicting that $W$ is strongly wandering.

Now we deal with the non-Feigenbaum case. Here $k_{1}=1,\left.f\right|_{W_{c_{1}}}$ is a diffeomorphism and $\partial W_{c_{1}} \cap U=\emptyset$. On the other hand, $U_{c_{1}} \subset \operatorname{cyc}(K)$, so $f\left(W_{c_{1}}\right) \subset \operatorname{cyc}(K)$ as well. Moreover, the choice of $\operatorname{cyc}(K)$ guarantees that the orbits of almost all its points accumulate on Crit. Hence $f\left(W_{c_{1}}\right)$ is the pairwise disjoint union (up to a measure zero set) of entry domains to $U$ and components of $U$. Since the density of $f^{j_{1}+1}(W)$ in $f\left(W_{c_{1}}\right)$ is $>1-\delta^{-1} \kappa^{2} \varepsilon_{0}$, it is also $>1-\delta^{-1} \kappa^{2} \varepsilon_{0}$ in one of these entry domains or components, call it $J_{2}$.

Now we repeat the argument. Say that $\left.\phi\right|_{J_{2}}=\left.f^{j_{2}}\right|_{J_{2}}, f^{j_{2}}\left(J_{2}\right)=U_{c_{2}}$, or $J_{2}$ is a component $U_{c_{2}}$ of $U$ (when we take $j_{2}=0$ ). Then $f^{j_{1}+j_{2}+1}(W)$ has density $>1-\delta^{-2} \kappa^{3} \varepsilon_{0}$ in $U_{c_{2}}$. If $c_{2}$ is a critical point of Feigenbaum type, then we get a contradiction as before. If not, then we find an entry domain to $U$ or a component of $U$, call it $J_{3}$, such that $f^{j_{1}+j_{2}+2}(W)$ has density $>1-\delta^{-2} \kappa^{4} \varepsilon_{0}$ in $J_{3}$. Proceeding in this way, we find intervals $J_{1}, J_{2}, \ldots, J_{d+1}$, each of them either an entry domain to $U$ or a component of $U$, say $f^{j_{i}}\left(J_{i}\right)=U_{c_{i}}$, such that the density of $f^{t_{i}}(W)$ in $U_{c_{i}}$ is $>1-\delta^{-(i-1)} \kappa^{2 i-1} \varepsilon_{0}$ for every $i=1,2, \ldots, d+1$, with $t_{i}=j_{1}+\cdots+j_{i}+i-1$. Find $i<i^{\prime}$ such that $c_{i}=c_{i^{\prime}}$. Then the densities of $f^{t_{i}}(W)$ and $f^{t_{i^{\prime}}}(W)$ in $U_{c_{i}}$ are $>1 / 2$, and we have the required contradiction.

\section{4. lim sup FULLnESS OF LEBESGUE MEASURE}

The following definition was first used in Barnes [Ba].

Definition 22. The system $(X, \mu, f)$ is called $\limsup$ full if $\limsup _{n \rightarrow \infty} \mu\left(f^{n}(A)\right)=1$ for any $A \in \mathcal{B}$ with $\mu(A)>0$.

Theorem 23. Let $f \in C_{\mathrm{nf}}^{3}(I)$ be a topologically mixing map having no Cantor attractors. Then $f$ is limsup full with respect to Lebesgue measure.

Proof. It suffices to show that if $f \in C_{\mathrm{nf}}^{3}(I)$ (and satisfying (11)) has an invariant interval $I^{\prime}$ such that $\left.f\right|_{I^{\prime}}$ is topologically mixing and has no Cantor attractors, then we have $\lim \sup _{n} \lambda\left(f^{n}(A)\right)=\lambda\left(I^{\prime}\right)$ for any measurable set $A \subset I^{\prime}$ of positive measure. 
Fix a critical point $c$ interior to $I^{\prime}$ and let $\varepsilon>0$ be small enough so that Theorem 21]holds and the corresponding interval $U_{c}$ lies in $I^{\prime}$. Let $x$ be a density point of $A$. Since $f$ has no Cantor attractors in $I^{\prime}$, there is no loss of generality in assuming $\omega(x)=I^{\prime}$ and (after replacing if necessary $A$ by some of its iterates) $x \in U_{c}$. Since all iterates of the induced map $F$ from Theorem 21 are well defined for $\lambda$-a.e. point in $U_{c}$, we can assume that this is the case for $x$. This implies that there are intervals $x \in H_{n} \subset U_{c}$ with $\bigcap_{n} H_{n}=\{x\}$ and integers $k_{n}$ such that $\left.f^{k_{n}}\right|_{H_{n}}: H_{n} \rightarrow U_{c^{\prime}}$ are diffeomorphisms with uniform distortion bound for some $c^{\prime} \in$ Crit' $^{\prime}$. Hence $\lambda\left(f^{k_{n}}\left(A \cap H_{n}\right)\right) \rightarrow \lambda\left(U_{c^{\prime}}\right)$ as $n \rightarrow \infty$. Observe that $U_{c^{\prime}} \subset I^{\prime}$, so there is $j$ such that $I^{\prime}=f^{j}\left(U_{c^{\prime}}\right)$ (because $\left.f\right|_{I^{\prime}}$ is topologically mixing). Then also $\lambda\left(f^{k_{n}+j}\left(A \cap H_{n}\right)\right) \rightarrow \lambda\left(I^{\prime}\right)$, as required.

Proposition 24. Let $f \in C_{\mathrm{nf}}^{3}(I)$ be topologically mixing. Then the following statements are equivalent:

(i) $f$ has an acip $\mu$;

(ii) $\liminf _{n} \lambda\left(f^{n}(A)\right)>0$ for every measurable set $A$ of positive Lebesgue measure;

(iii) $\liminf _{n} \lambda\left(f^{n}(A)\right)=1$ for every measurable set $A$ of positive Lebesgue measure.

In this case $\mu$ is equivalent to $\lambda$ (that is, $\mu(A)$ if and only if $\lambda(A)=0$ ) and $\lambda_{2}$ is ergodic and conservative.

Remark 25. Under $C^{3}$ assumptions, no transitive Cantor set can have positive measure, [SV, Theorem E(1), cf. also Remark 1], so in particular a Cantor attractor has Lebesgue measure 0 , and cannot support an acip. We expect this to be true in the $C^{2}$ setting as well, but we have no proof.

Proof. The implication (iii) $\Rightarrow$ (ii) is trivial.

We prove (ii) $\Rightarrow(\mathrm{i})$. According to [St], the existence of an acip for $f$ is equivalent to the existence of $\delta>0$ and $0<\alpha<1$ such that $\lambda(A)<\delta$ implies $\lambda\left(f^{-n}(A)\right)<\alpha$ for every $n$ and $A$. Hence, if $f$ does not admit an acip, then there are sets $\left\{A_{k}\right\}$ and integers $\left(n_{k}\right)_{k \geq 1}$ with $n_{k} \rightarrow \infty, \lambda\left(A_{k}\right) \rightarrow 0$ and $\lambda\left(f^{-n_{k}}\left(A_{k}\right)\right) \geq 1-2^{-k-1}$. Take $A=\bigcap_{k} f^{-n_{k}}\left(A_{k}\right)$, then $\lambda(A) \geq \frac{1}{2}$ and $\liminf _{n} \lambda\left(f^{n}(A)\right)=0$, contrary to condition (ii).

For the implication (i) $\Rightarrow($ iii), assume that $f$ admits an acip $\mu$. Since (wild) Cantor attractors have zero Lebesgue measure, $\mu$ cannot be supported on them. Then Theorem 23 applies.

We claim that $\mu$ is equivalent is to $\lambda$. Assume the contrary to find a measurable set $A$ such that $\mu(A)=0<\lambda(A)$. Let $B=\bigcup_{n=0}^{\infty} f^{-n}(A)$. Then $\mu(B)=0$ but, by Theorem 23, $\lambda(B)=1$. This is impossible.

The equivalence of $\mu$ and $\lambda$ (or just the absolute continuity of $\mu$ ) and Theorem 23 imply that if $\mu(A)>0$, then $\limsup _{n} \mu\left(f^{n}(A)\right)=1$. Since $\mu$ is invariant, the sequence $\left\{\mu\left(f^{n}(A)\right)\right\}_{n \in \mathbb{N}}$ is non-decreasing. Therefore $\lim _{n} \mu\left(f^{n}(A)\right)=1$, and also $\lim _{n} \lambda\left(f^{n}(A)\right)=$ 1 by the equivalence of $\mu$ and $\lambda$. Moreover, $\lambda$ is exact, hence $\lambda_{2}$ is ergodic (Lemma [18), and $\mu_{2}$ is conservative (because of Poincaré recurrence), so $\lambda_{2}$ is conservative as well (because $\mu_{2}$ and $\lambda_{2}$ are equivalent). 


\section{LI-YORKE PAIRS AND SCRAMBLED SETS}

In this section we prove our results on scrambled sets and the $\lambda_{2}$-measure of the sets Dis, Asymp and LY in the case that $f$ had no wild attractor.

Proof of Theorem B. Assume that a scrambled set $S$ has positive measure. We can assume that all its points are attracted by the same attractor, which must be either a cycle of intervals containing a dense orbit, or a minimal Cantor set. Since $f^{n}$ is one-to-one on $S$ for every $n$, the first possibility can be immediately discarded by Theorem 22 . Hence we may assume that all points of $S$ are attracted by a minimal Cantor set $W$. (In fact $W$ must be a wild attractor, because points attracted by solenoidal sets are approximately periodic and a scrambled set can contain at most one approximately periodic point by Proposition 6.)

By Theorem $\mathrm{A}$ there are integers $n<m$ such that $f^{n}(S) \cap f^{m}(S) \neq \emptyset$. Let $x \in f^{n}(S) \cap$ $f^{m}(S)$. Then there is $y \in f^{n}(S)$ such that $f^{m-n}(y)=x$. Since $(x, y)$ is a Li-Yorke pair, so is $\left(f^{m-n}(x), f^{m-n}(y)\right)=\left(f^{m-n}(x), x\right)$. Find a sequence $\left\{l_{k}\right\}$ such that $\mid f^{m-n+l_{k}}(x)-$ $f^{l_{k}}(x) \mid \rightarrow 0$. We may assume that $\left(f^{l_{k}}(x)\right)_{k \in \mathbb{N}}$ accumulates at $p \in W$. Then $f^{m-n}(p)=p$, which is impossible because $W$ is infinite and minimal.

Proposition 26. A multimodal map $f$ has no closed invariant scrambled set (apart from a singleton set).

Proof. Suppose by contradiction that the closed non-singleton $S$ is invariant and scrambled. Then clearly it can contain at most one fixed point. Let $y \in S$ be a nonfixed point. Then because $(y, f(y))$ is Li-Yorke, there is a sequence $\left(n_{k}\right)_{k \in \mathbb{N}}$ such that $\lim _{k}\left|f^{n_{k}}(y)-f^{n_{k}}(f(y))\right|=0$ and $\left\{f^{n_{k}}(y)\right\}$ converges. By continuity $\lim _{k} f^{n_{k}}(y)=$ $\lim _{k} f^{n_{k}+1}(y)=f\left(\lim _{k} f^{n_{k}}(y)\right)$, so the limit is a fixed point $p \in S$. Since $(y, f(y))$ is Li-Yorke, $\left\{f^{n}(y)\right\}$ does not converge to $p$.

Since $f$ is multimodal, there are a sequence $\left(m_{k}\right)$ and a number $\varepsilon>0$ such that $f^{m_{k}}(y) \rightarrow p$ but $\left|f^{m_{k}-1}(y)-p\right|>\varepsilon$. By taking a subsequence, we can assume that $\left\{f^{m_{k}-1}(y)\right\}_{k \in \mathbb{N}}$ converges, say to $q$. But then $\left.p=\lim _{k} f^{m_{k}}(y)=f\left(\lim _{k} f^{m_{k}}(y)\right)\right)=f(q)$, so $(p, q)$ is not Li-Yorke. This contradiction proves the proposition.

Proof of Theorem $\left[\boldsymbol{C}\right.$. Assume that $\lambda_{2}$ (Asymp $\backslash($ AsPer $\times$ AsPer $\left.)\right)>0$. Then there are a point $x \in I \backslash$ AsPer and a Borel set $Y \subset I \backslash$ AsPer of positive measure such that $\lim _{n \rightarrow \infty}\left|f^{n}(x)-f^{n}(y)\right|=0$ for every $y \in Y$. We can assume that $\operatorname{orb}(x)$ accumulates on a non-periodic point $u$.

For every $n \geq 0$, let $d_{n}: Y \rightarrow \mathbb{R}$ be defined by $d_{n}(y)=\sup _{m \geq n}\left|f^{m}(x)-f^{m}(y)\right|$. Then $\left(d_{n}\right)$ is a sequence of Borel measurable maps converging pointwise to zero. According to Egorov's theorem we can remove from $Y$ a small set (so that the remaining set $Z$ has positive measure) in such a way that $\left\{\left.d_{n}\right|_{Z}\right\}_{n \in \mathbb{N}}$ converges uniformly to zero, that is, $\operatorname{diam}\left(f^{n}(Z)\right) \rightarrow 0$ as $n \rightarrow \infty$.

Use Theorem $\mathrm{A}$ to find integers $k>m$ such that $f^{k}(Z) \cap f^{m}(Z) \neq \emptyset$. Recall that orb $(Z)$ accumulates at a point $u$ with $\left|f^{j}(u)-u\right|=\varepsilon>0$ for $j=k-m$. Find $\delta \in(0, \varepsilon / 4)$ such that $\left|f^{j}(v)-f^{j}(w)\right|<\varepsilon / 2$ whenever $|v-w|<2 \delta$. Next take $l>m$ so that $\operatorname{dist}\left(f^{l}(Z), u\right)<\delta$ 
and $\operatorname{diam}\left(f^{l}(Z)\right)<\delta$. Then $\left.\mid f^{l}(z)-u\right) \mid<2 \delta<\varepsilon / 2$, hence $\left|f^{l+j}(z)-f^{j}(u)\right|<\varepsilon / 2$ for every $z \in Z$. Thus $f^{l+j}(Z) \cap f^{l}(Z)=\emptyset$, contradicting $f^{m+j}(Z) \cap f^{m}(Z) \neq \emptyset$ and $l>m$.

Proposition 27. If $(I, \mu, f)$ is exact, then $\mu_{2}($ Dis $)=0$.

Proof. Assume by contradiction that $\mu_{2}$ (Dis) $>0$. Write $\operatorname{Dis}_{x}=\{y \in I:(x, y)$ is distal $\}$ and $G_{\text {Dis }}:=\left\{x: \mu\left(\operatorname{Dis}_{x}\right)>0\right\}$. Then by Fubini's Theorem, $\mu\left(G_{\text {Dis }}\right)>0$. For $x \in G_{\text {Dis }}$, take

$$
Y_{\varepsilon}:=\left\{y \in \operatorname{Dis}_{x}: \liminf _{n \rightarrow \infty}\left|f^{n}(x)-f^{n}(y)\right| \geq \varepsilon\right\} .
$$

Clearly $Y_{\delta} \subset Y_{\varepsilon}$ if $\delta>\varepsilon$ and $f^{n}\left(Y_{\varepsilon}\right) \cap f^{n}\left(I \backslash Y_{\varepsilon}\right)=\emptyset$ for all $n \geq 0$. If for some $\varepsilon>0$, both $Y_{\varepsilon}$ and $I \backslash Y_{\varepsilon}$ have positive measure, then we have a contradiction to exactness. The remaining possibility is that there is $\varepsilon=\varepsilon(x)>0$ such that $Y_{\varepsilon}$ has full measure, whereas $\lambda\left(Y_{\delta}\right)=0$ for all $\delta>\varepsilon$. Now take $\eta>0$ such that $G_{\eta}:=\left\{x \in G_{\text {Dis }}: \varepsilon(x)>\eta\right\}$ has positive measure. Let $R>1 / \eta$, and take distinct points $x_{0}, x_{1}, \ldots, x_{R} \in G_{\eta}$. Clearly these points can be chosen so that $\liminf _{n}\left|f^{n}\left(x_{i}\right)-f^{n}\left(x_{j}\right)\right|>\eta$ for all $i \neq j$. Take $N$ minimal such that $\left|f^{n}\left(x_{i}\right)-f^{n}\left(x_{j}\right)\right|>\eta$ for all $i \neq j$ and $n \geq N$. However, by the choice of $R$, there is no space in $I$ to fit the points $f^{N}\left(x_{i}\right), i=0, \ldots, R$, so that they have pairwise distance greater than $\eta$. This contradiction proves that $\mu_{2}(\mathrm{Dis})=0$.

Proof of Theorem $\boldsymbol{D}$. Theorem 23 implies that $f$ is limsup full w.r.t. Lebesgue measure. By [Ba, Theorem A], if $f: I \rightarrow I$ is a multimodal surjective map that is lim sup full with respect to a measure $\mu$, then $f$ is exact. (The proof is stated for $d$-to- 1 maps, but applies with minor changes to the "at most $d$-to- 1 " setting as well.) Hence $\lambda$ is exact. By Lemma 18, $\lambda_{2}$ is ergodic for $\left(I^{2}, f_{2}\right)$.

Take $x \in I$. We prove that there is a full measure set $A_{x}$ such that $\lim _{\sup } \operatorname{sum}_{n \rightarrow \infty} \mid f^{n}(y)-$ $f^{n}(x) \mid \geq \operatorname{diam}(I) / 2$ for every $y \in A_{x}$. If the opposite is true, then there are a set $A$ of positive measure and an $m \in \mathbb{N}$ such that $\left|f^{n}(y)-f^{n}(x)\right|<\operatorname{diam}(I) / 2$ for every $y \in A$ and every $n \geq m$. But this contradicts the conclusion of Theorem [23] applied to $A$, i.e., that $\lim \sup _{n} \lambda\left(f^{n}(A)\right)=1$. Similarly, we can prove that there is a full measure $B_{x}$ such that $\limsup _{n \rightarrow \infty}\left|f^{n}(y)-f^{n}(x)\right|=0$ for every $y \in B_{x}$. Indeed, if this were false then there are $m \in \mathbb{N}, \varepsilon>0$ and a set $B$ of positive measure such that $\left|f^{n}(y)-f^{n}(x)\right|>\varepsilon$ for every $y \in A$ and every $n \geq m$. Again, this contradicts that $\lim _{\sup _{n}} \lambda\left(f^{n}(B)\right)=1$. Hence

$$
\liminf _{n \rightarrow \infty}\left|f^{n}(y)-f^{n}(x)\right|=0, \quad \limsup _{n \rightarrow \infty}\left|f^{n}(y)-f^{n}(x)\right| \geq \operatorname{diam}(I) / 2,
$$

for every $y \in U_{x}=A_{x} \cap B_{x}$.

By Proposition 24 and Lemma 19 we immediately recover a well-known result on weakmixing.

Corollary 28. If $f \in C_{\mathrm{nf}}^{3}(I)$ is topologically mixing and has an acip, then $\lambda_{2}$-a.e. $(x, y)$ has a dense orbit in $I^{2}$.

Remark 29. Probably the earliest result in this direction dates back to Ledrappier who proves in [Le, Theorem 1] that a certain class of interval maps is weak Bernoulli. Keller [Ke] proved (weak-)mixing for acips $\mu$ of multimodal maps assuming negative Schwarzian derivative. he also showed that $d \mu / d \lambda$ is bounded away from zero on $\operatorname{supp}(\mu)$. One can 
prove that $\mu$ is also mixing by means of an induced map $F: U \rightarrow U$ as in Theorem [21] which possesses an acip $\nu$, cf. [Y]. In fact, by taking an appropriate power of $F^{N}, U$ decomposes into a finite number of $F^{N}$-invariant parts on which $\nu$ is invariant and mixing. Pulling back $\nu$ to the original system, we recover $\mu$ and by the topologically mixing condition, $\mu$ can have only one mixing component.

Conservativity of $\lambda_{2}$ is crucial in Lemma 19, and even if $\lambda$ itself is conservative for $(I, f)$, this does not guarantee that $\lambda_{2}$ is conservative for the Cartesian product. It is for this reason that Proposition 27 and Theorem D are not just direct consequences of ergodicity of $\lambda_{2}$ from Lemma 18. The following conjecture suggests conditions under which $\lambda_{2}$-a.e. pair is Li-Yorke, but has no dense orbit.

Conjecture 30. We think that if $(I, f, \lambda)$ is conservative and has no acip, but instead the induced time $k_{x}$ associated to the induced map in Theorem 21 is non-integrable w.r.t. Lebesgue, and in fact the tail $\lambda\left(\left\{x: k_{x}>s\right\}\right) \geq 1 / \log s$, then the product system $\left(I^{2}, f_{2}, \lambda_{2}\right)$ is dissipative.

\section{Li-Yorke Chaos in the PREsence of CANTOR ATtractors}

In this section, we concentrate on $C^{2}$ unimodal maps with Cantor attractors $\mathcal{A}=\omega(c)$ for the unique critical point $c$. If $f$ is infinitely renormalizable (i.e., type (3) in Proposition 9), then the situation regarding Li-Yorke pairs is well-known: there are none. Instead, the attractor is Lyapunov stable and conjugate to an adding machine $(\Omega, g)$. In other words, $\Omega=\left\{\left(\omega_{j}\right)_{j \geq 1}: 0 \leq \omega_{j}<p_{j}\right\}$, for some sequence $\left(p_{i}\right)_{i \geq 1}$ of integers $p_{i} \geq 2$ (where $p_{1} \cdots p_{i}$ are the periods of the periodic intervals) such that is equipped with product topology and the map $g$ of "adding 1 and carry":

$$
g\left(\omega_{1}, \omega_{2}, \ldots\right)= \begin{cases}0,0, \ldots, 0, \omega_{k}+1, \omega_{k+1}, \omega_{k+2}, \ldots & \text { if } k=\min \left\{i: \omega_{i}<p_{i}-1\right\} \\ 0,0,0,0, \ldots & \text { if } \omega_{i}=p_{i}-1 \text { for all } i \geq 1\end{cases}
$$

The following classification is due to [BrJ, Proposition 5.1].

Proposition 31. Let $f: I \rightarrow I$ be a continuous map and $x \in I$. The system $(\omega(x), f)$ is conjugate to some $\left(p_{i}\right)$-adic adding machine if and only if $x$ is approximately but not asymptotically periodic, see Definition 1 .

However, there are several constructions leading to strange adding machines, i.e., (critical) omega-limit sets that are conjugate to adding machines, but not involving periodic intervals, see [BKM, BrJ, $[\mathrm{Br} 4]$. In [Br4] it is shown that a strange adding machine can still be an attractor, but in this case it is a wild and not a solenoidal attractor.

The dynamics on such attractors can frequently be understood in terms of generalized adding machines as done in [BKS] and in the proof of Theorem 34 below. Such generalized adding machines are based on the sequence of cutting time, which we will define now.

Let $Z_{n}(x)$ be the $n$-cylinder, i.e., maximal interval containing $x$ on which $f^{n}$ is monotone. Unless $x \in \cup_{m<n} f^{-m}(c), Z_{n}(x)$ is well-defined, but the critical point itself has two sets $Z_{n}^{ \pm}(c)$ on either side of it, with $\mathcal{D}_{n}:=f^{n}\left(Z_{n}^{ \pm}(c)\right)$ independent of \pm . One can show that for every $x$ and $n \geq 1$, there is $m \leq n$ such that $f^{n}\left(Z_{n}(x)\right)=\mathcal{D}_{m}$; if $x \in f^{-m}(c)$, then 
$x$ is the common boundary point of two $n$-cylinder sets $Z_{n}^{ \pm}(x)$, but $f^{n}\left(Z_{n}^{ \pm}(x)\right)=\mathcal{D}_{m}$ independently of \pm . We say that $n$ is a cutting time if $\mathcal{D}_{n} \ni c$, and we list them in increasing order $1=S_{0}<S_{1}<\ldots$. Write $c_{n}:=f^{n}(c)$. By a short induction proof one can show that $\mathcal{D}_{1}=\left[0, c_{1}\right]$ and for $n \geq 2$

$$
\mathcal{D}_{n}=\left[c_{n}, c_{\beta(n)}\right] \text { and } \mathcal{D}_{n} \subset \mathcal{D}_{\beta(n)},
$$

where the map $\beta: \mathbb{N} \rightarrow \mathbb{N}$ is defined as $\beta(n)=n-\max \left\{S_{k}: S_{k}<n\right\}$. In the special case that $n=S_{k}$ is a cutting time, this means that $S_{k}-S_{k-1}$ is again a cutting time, so one can define the kneading map $Q: \mathbb{N} \rightarrow \mathbb{N} \cup\{0, \infty\}$ by

$$
S_{k}=S_{k-1}+S_{Q(k)},
$$

see $\left[\mathrm{H},[\mathrm{Br} 2]\right.$. Here $Q(k)=\infty$ means that $S_{k}$ does not exist; in this case $c$ is attracted to an orbit of period $S_{k-1}$ or $2 S_{k-1}$. Since we assumed that $f$ has a Cantor attractor, $Q(k)<\infty$ for all $k \in \mathbb{N}$. Some properties of the kneading map related to $\omega(c)$ are as follows.

Proposition 32. (a) The minimal n such that $Z_{n}^{ \pm}(c) \subset\left[c, c_{S_{k}}\right]$ is $n=S_{Q(k+1)}$.

(b) If $f$ is a unimodal map with kneading map $Q(k) \rightarrow \infty$, then the length $\left|\mathcal{D}_{n}\right| \rightarrow 0$ and $\omega(c)$ is a minimal Cantor set.

(c) If $\max _{k} k-Q(k) \leq B$, then $\omega(c) \subset \cup_{n=1+S_{k}}^{S_{k+B}} \mathcal{D}_{n}$ for every $k$.

(d) The map is renormalizable if and only if there is $k \geq 1$ such that $k=Q(k+1) \leq$ $Q(k+j)$ for all $j>1$. In this case $S_{k}$ is the period of renormalisation.

Proof. By definition of the kneading map, $n=S_{Q(k+1)}$ is the smallest positive iterate such that $f^{n}\left(\left[c, c_{S_{k}}\right]\right) \ni c$, implying statement (a).

Under the assumption that $Q(k) \rightarrow \infty$, this means that $\left|c_{S_{k}}-c\right| \rightarrow 0$ and also $\left|c_{S_{Q(k)}}-c\right| \rightarrow$ 0 as $k \rightarrow \infty$. Since $\mathcal{D}_{S_{k}}=\left[c_{S_{k}}, c_{S_{Q(k)}}\right]$, the non-Contraction Principle shows that $\left|\mathcal{D}_{n}\right| \rightarrow 0$ as well. Minimality of $\omega(c)$ follows as in i.e., [Br2, Proposition 2] (which proves that $c$ is persistently recurrent) and [Br2, Lemma 8]. This proves statement (b).

For statement (c), observe that $\mathcal{D}_{1+S_{l}} \subset \mathcal{D}_{1+S_{k}}$ for all $l \geq k+B$ because of the assumption $\max _{k}\{k-Q(k)\} \leq B$ and statement (a). It follows that $\mathcal{D}_{m+S_{k+B}} \subset \mathcal{D}_{m+S_{k}}$ for all $m \geq 1$, and $\cup_{n \geq 1+S_{k}} \mathcal{D}_{n} \subset \cup_{n=1+S_{k}}^{S_{k+B}} \mathcal{D}_{n}$. By definition, $\omega(c) \subset \mathrm{Cl} \cup_{n \geq 1+S_{k}} \mathcal{D}_{n} \subset \mathrm{Cl} \cup_{n=1+S_{k}}^{S_{k+B}} \mathcal{D}_{n}=$ $\cup_{n=1+S_{k}}^{S_{k+B}} \mathcal{D}_{n}$ as asserted.

Part (d) is [Br2, Proposition 1(iii)].

Special types of unimodal maps are the Feigenbaum map $\left(S_{k}=2 S_{k-1}\right)$ and the Fibonacci map $\left(S_{k}=S_{k-1}+S_{k-2}\right)$. We call $f$ Fibonacci-like if $\{k-Q(k)\}_{k}$ is bounded.

The proof of the existence of wild attractors was first established in [BKNS] for Fibonacci maps with sufficiently critical order $\ell$. In [Br3], this result was extended to $C^{3}$ unimodal maps with negative Schwarzian derivative, sufficiently large critical order and eventually non-decreasing kneading map $Q$ such that $\limsup _{k} k-Q(k) \leq B$. If the cutting times increase more slowly than Fibonacci-like, then no unimodal map with finite critical order can have a wild attractor.

Before we continue, let us give a short exposition how the existence of wild attractor is proved for unimodal maps. There are two approaches, both based on a random walk on 
a Markov graph. In [BKNS, Br3, BHa], this Markov graph of $I$ is based on preimages of the orientation reversing fixed point $p$. The states of that Markov system are pairs of intervals $U_{k} \subset\left(Z_{S_{k}}^{+}(c) \cup Z_{S_{k}}^{-}(c)\right) \backslash\left(Z_{S_{k+B}}^{+}(c) \cup Z_{S_{k+B}}^{-}(c)\right)$ and $\partial U_{k}$ belongs to the backward orbit of $p$. The induced Markov map defined by $\left.G\right|_{U_{k}}=f^{S_{k}}$ preserves the partition $\left\{U_{k}\right\}$ of $[\hat{p}, p]$, where $f^{-1}(p)=\{p, \hat{p}\}$. Viewing the dynamics of $G$ as a random walk, we define "random variables" $\chi_{n}$ by

$$
\chi_{n}(x)=k \text { if } G^{n}(x) \in U_{k} .
$$

It is then shown that this process has positive drift, i.e., the expectations (measured with respect to Lebesgue measure)

$$
\mathbb{E}\left(\chi_{n+1}-k \mid \chi_{n}=k\right) \geq \eta>0
$$

uniformly in $n$ and $l$. The second moments $\mathbb{E}\left(\left(\chi_{n+1}-k\right)^{2} \mid \chi_{n}=k\right)$ are shown to be bounded as well. It follows that $\chi_{n}(x) \rightarrow \infty$ for $\lambda$-a.e. $x$ and hence $G^{n}(x) \rightarrow c$. Since $U_{k} \subset\left(Z_{S_{k}}^{+}(c) \cup Z_{S_{k}}^{-}(c)\right), f^{S_{k}}\left(U_{k}\right) \subset \mathcal{D}_{S_{k}}$ and since $\left|\mathcal{D}_{n}\right| \rightarrow 0$ as $n \rightarrow \infty$, this means for the original map that $f^{n}(x) \rightarrow \omega(c)$ for $\lambda$-a.e. $x$, so $\mathcal{A}=\omega(c)$ is an attractor.

In [BHa, Theorem 5.2], a further conclusion is drawn from the positive drift, namely a Borel-Cantelli Lemma argument shows that for $\lambda$-a.e. $x \in \operatorname{Bas}(\mathcal{A})$, there is $k_{0}=k_{0}(x)$ such that such that for all $k \geq k_{0}$

$$
\text { if } G^{m}(x) \in U_{k} \text {, then } G^{m+j}(x) \notin U_{k} \text { for } j>k \text {. }
$$

In this paper, we will use a second approach from [Br1, BKS], where the Markov system is a disjoint union $\hat{I}=\sqcup_{n \geq 2} \mathcal{D}_{n}$. (the Hofbauer tower) for intervals $\mathcal{D}_{n}$ defined above. Let $\hat{f}: \hat{I} \rightarrow \hat{I}$ be defined by

$$
\hat{f}\left(\mathcal{D}_{n}\right)= \begin{cases}\mathcal{D}_{n+1} & \text { if } n \text { is not a cutting time; } \\ \mathcal{D}_{1+S_{k}} \sqcup\left(\mathcal{D}_{1+S_{Q(k)}} \backslash\left\{c_{1}\right\}\right) & \text { if } n=S_{k} \text { is a cutting time. }\end{cases}
$$

Then $i \circ \hat{f}=f \circ i$, where $i: \hat{I} \rightarrow I$ is the inclusion map, and $f$ is continuous, except at the points $c \in \mathcal{D}_{S_{k}}, k \geq 1$, which are mapped to $c_{1} \in \mathcal{D}_{1+S_{k}}$. Since these are only countable many points, this has no effect on the Lebesgue typical behavior. The collection $\left\{\mathcal{D}_{n}\right\}_{n \geq 2}$ is a Markov partition of $\hat{I}$, because $\hat{f}$ maps each $\mathcal{D}_{n}$ to the union of partition elements (ignoring again the point $c_{1} \in \mathcal{D}_{1+S_{Q(k)}}$ ). Starting in some $\mathcal{D}_{n}$, the subsequence intervals visited are unique determined up to the moment we reach some $\mathcal{D}_{S_{k}}$, where we have a choice between $\mathcal{D}_{1+S_{k}}$ and $\mathcal{D}_{1+S_{Q(k)}}$. Therefore it suffices to consider the transitions from interval $E_{l}:=\mathcal{D}_{1+S_{l}}$ realized by the $S_{Q(l+1)}$-th image of $f$, see Figure 1. It follows that $\left\{E_{l}\right\}_{l \geq 1}$ is a Markov partition for the induced map

$$
F: \sqcup_{l \geq 0} E_{l} \rightarrow \sqcup_{l \geq 0} E_{l},\left.\quad F\right|_{E_{l}}=\hat{f}^{S_{Q(l+1)}} .
$$

One can show that if $f$ is renormalizable of period $S_{k}$, then $\sqcup_{l \geq k-1} E_{l}$ is a trapping region for $F$, see part (d) of Proposition 32. Now we need to translate the results on positive drift to the current set-up. Write $\hat{\chi}_{n}(y)=l$ if $F^{n}(y) \in E_{l}$.

Lemma 33. If $\left\{\chi_{n}\right\}_{n \geq 0}$ has positive drift as in (6) (and bounded second moments), then for $\lambda$-a.e. $y \in \sqcup_{l \geq 1} E_{l}$, we have $\hat{\chi}_{n}(y) \rightarrow \infty$. Furthermore, there is $\hat{k}_{0}=\hat{k}_{0}(y)$ and $C>0$ such that for all $\hat{k} \geq \hat{k}_{0}$, if $\hat{\chi}_{n}(y)=\hat{k}$, then $\hat{\chi}_{n+j}(y)>\hat{k}$ for all $j>C \hat{k}$. 


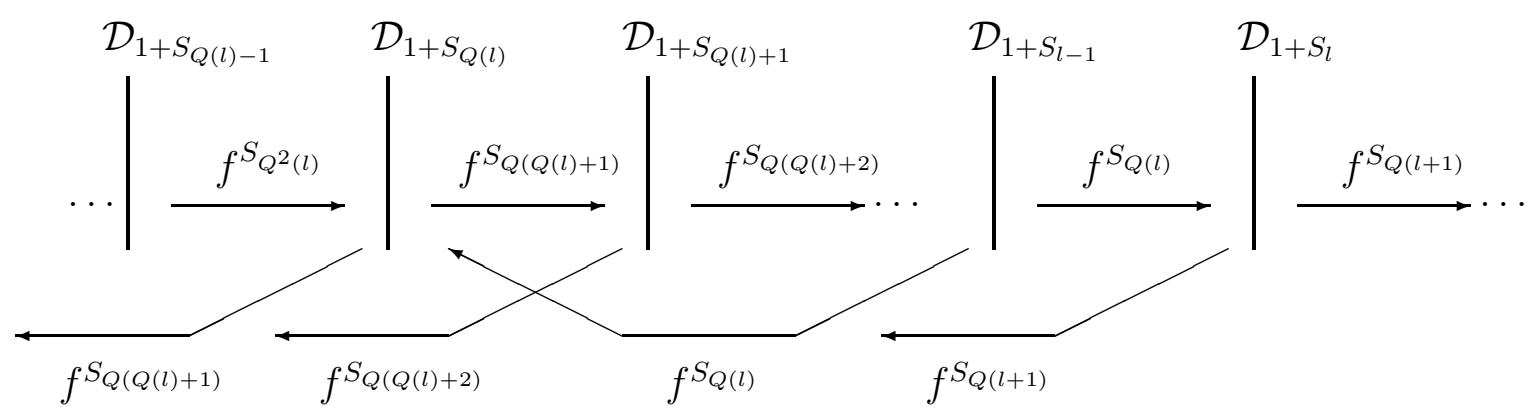

Figure 1. The transitions from $\mathcal{D}_{1+S_{l}}, l \geq 1$. Backward transitions go from $\mathcal{D}_{1+S_{l}}$ to $\mathcal{D}_{1+S_{Q(l+1)}}$.

Proof. The positive drift of $\left\{\chi_{n}\right\}$ ensures that for $\lambda$-a.e. $x$ and $k \in \mathbb{N}$, there is $n_{k}$ such that $G^{n}(x) \in \cup_{j \geq k} U_{j}$ for all $n \geq n_{k}$. Since $U_{k} \subset Z_{S_{k}}^{+}(c) \cup Z_{S_{k}}^{-}(c)$, the image $f^{S_{k}}\left(U_{k}\right) \subset \mathcal{D}_{S_{k}}$ and $f^{1+S_{k}}\left(U_{k}\right) \subset \mathcal{D}_{1+S_{k}} \cup \mathcal{D}_{1+S_{Q(k)}}=E_{k} \cup E_{Q(k)}$. This means that $y:=f^{1+S_{k}} \circ G^{n}(x) \in$ $E_{k} \cup E_{Q(k)}$. If consequently $G^{n+1}(x) \in U_{l}, l>k$, then $f^{S_{l}}(y) \in E_{l} \cup E_{Q(l)}$ but the move from $E_{k}$ or $E_{Q(k)}$ up to $E_{l}$ or $E_{Q(l)}$ in $\sqcup_{j \geq 0} E_{j}$ involves passages through the intermediate states $E_{i}$ as well, but "lower" states $E_{i}, i<k-B \leq \min _{j \geq k} Q(j)$ are avoided. Therefore $\hat{\chi}_{n}(f(x)) \rightarrow \infty$ for all $n \rightarrow \infty$.

For the second statement, observe that the passage from $E_{k}$ or $E_{Q(k)}$ up to $E_{l}$ or $E_{Q(l)}$ requires $u_{n}$ iterates of $F$ for some $l-k-B \leq u_{n} \leq l-k+B$. Suppose $G^{m}(x) \in U_{k}$, $k \geq k_{0}(x)$ as in (7), then $G^{m+j}(x) \notin U_{k}$ for $j \geq k$. Take $\hat{k} \in\{k, Q(k)\}$ such that $y=f^{1+S_{k}}\left(G^{m}(x)\right) \in E_{\hat{k}}$. The iterates $m+1, \ldots, m+k$ of $G$ correspond to $\sum_{j=m+1}^{m+k} u_{j}$ iterates of $F$. Each $u_{j} \geq 1$, and if $\chi_{m+j}(x)<\chi_{m+j-1}(x)$, then this single iterate of $G$ corresponds to a single iterate of $F$, reducing the index of the state by at most $B$. If $\chi_{m+j}(x) \gg \chi_{m+j-1}(x)$, then one iterate of $G$ corresponds to many iterates of $F$, but if some of these iterates brings $y$ above state $E_{\hat{k}+k B}$, then it would take more than $k$ steps of $G$ to return, hence this will not occur. Thus, if the $\sum_{j=m+1}^{m+\hat{k}} u_{j}$ iterates of $F$ (corresponding $k$ iterates of $G$ ) keep $y$ close to state $E_{\hat{k}}$, then $\sum_{j=m+1}^{m+\hat{k}} u_{j} \leq 2 B k \leq 3 B \hat{k}$, where the last inequality follows because $k-B \leq \hat{k} \leq k$. This proves the second statement for $C=3 B$.

The dynamics of wild attractors has been investigated in [BKS]. In that paper, various combinatorial types are presented for which $(\mathcal{A}, f)$ is semi-conjugate to a monothetic group $(G, g)$, where $g: G \rightarrow G$ is an isometry for which every orbit is dense. The best known example goes back to Lyubich and Milnor [LM]; it is the Fibonacci map and its omega-limit set $\omega(c)$ factorizes over the golden mean circle rotation. In [BKS], similar examples are shown factorising onto tori of any dimension, and even onto a solenoid. On the other hand, BKS] gives examples for which $(\mathcal{A}, f)$ is weak mixing with respect to the unique invariant probability measure $\mu$ supported on $\mathcal{A}$. In [BHa], the simplest such example is shown to be Lebesgue exact as well.

Let $\mathfrak{f} \mathfrak{p}(x):=x-\operatorname{round}(x) \in\left[-\frac{1}{2}, \frac{1}{2}\right)$ be the signed distance of $x$ to the nearest integer. 
Theorem 34. Assume that a unimodal map has a wild attractor with positive drift. If there exists $\rho$ such that the cutting times satisfy

$$
\sum_{k} k \max _{i \geq k-B}\left|\mathfrak{f} \mathfrak{p}\left(\rho S_{k}\right)\right|<\infty,
$$

for $B=\lim \sup _{k} k-Q(k)$, then $\lambda_{2}($ Dis $)=1$.

\section{Proof. Step 1: Construction of the factor map.}

An enumeration scale is a symbolic system resembling an adding machine as in (4) based on, in this case, the sequence of cutting times. Any non-negative integer $n$ can be written in a canonical way as a sum of cutting times: $n=\sum_{j} e_{j} S_{j}$, where

$$
e_{j}:= \begin{cases}1 & \text { if } j=\max \left\{k ; S_{k} \leq n-\sum_{m>k} e_{m} S_{m}\right\} \\ 0 & \text { otherwise. }\end{cases}
$$

In particular $e_{j}=0$ if $S_{j}>n$. In this way we can code the non-negative integers as zero-one sequences with a finite number of ones: $n \mapsto\langle n\rangle \in\{0,1\}^{\mathbb{N}}$. Let $E_{0}=\langle\mathbb{N} \cup\{0\}\rangle$ be the set of such sequences, and let $E$ be the closure of $E_{0}$ in the product topology. This results in

$$
E:=\left\{e \in\{0,1\}^{\mathbb{N}} ; e_{i}=1 \Rightarrow e_{j}=0 \text { for } Q(i+1) \leq j<i\right\} .
$$

The condition in this set follows because if $e_{i}=e_{Q(i+1)}=1$, then this should be rewritten to $e_{i}=e_{Q(i+1)}=0$ and $e_{i+1}=1$. It follows immediately that for each $e \in E$ and $j \geq 0$,

$$
e_{0} S_{0}+e_{1} S_{1}+\cdots+e_{j} S_{j}<S_{j+1} .
$$

We denote by $g$ the standard addition of 1 by means of "add and carry", cf. (4). Let $\langle n\rangle$ be the representation of $n \in \mathbb{N} \cup\{0\}$ in the enumeration scale based on $\left\{S_{k}\right\}_{k \geq 0}$. Obviously $g(\langle n\rangle)=\langle n+1\rangle$. Under the condition that $Q(k) \rightarrow \infty, g: E \rightarrow E$ is continuous, and is invertible on $E \backslash\{\langle 0\rangle\}$, see [BKS, GLT]. Since $\sum_{k}\left|\mathfrak{f} \mathfrak{p}\left(\rho S_{k}\right)\right|<\infty$, we can define a continuous projection $\pi_{\rho}: E \rightarrow \mathbb{S}^{1}$ by

$$
\pi_{\rho}(e)=\sum_{k} e_{k} \mathfrak{f} \mathfrak{p}\left(\rho S_{k}\right) \bmod 1,
$$

and $\pi_{\rho} \circ g=R_{\rho} \circ \pi_{\rho}$ for the circle rotation $R_{\rho}: x \mapsto x+\rho \bmod 1$. At the same time, the map $P: E \rightarrow \mathcal{A}$ defined as the continuous extension of $P(\langle n\rangle)=f^{n}(c)$ satisfies $P \circ g=f \circ P$. We know from [BKS] that there is semi-conjugacy $\pi=\pi_{\rho} \circ P^{-1}: \mathcal{A} \rightarrow \mathbb{S}^{1}$

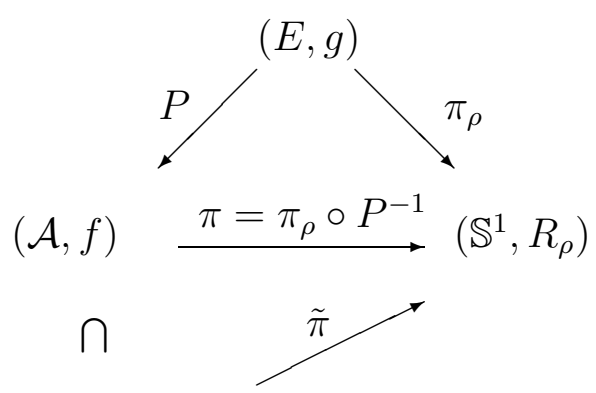

$(\operatorname{Bas}(\mathcal{A}), f)$ 
such that $\pi \circ f=R_{\rho} \circ \pi$, provided $\sum_{k}\left|\mathfrak{f} \mathfrak{p}\left(\rho S_{k}\right)\right|<\infty$. A more direct way to construct $\pi: \mathcal{A} \rightarrow \mathbb{S}^{1}$ is by setting

$$
\pi(x)=\left\{\begin{aligned}
\rho n \bmod 1 & \text { if } x=c_{n}, \\
\lim _{j \rightarrow \infty} \rho n_{j} \bmod 1 & \text { if } x \in \mathcal{A} \backslash \operatorname{orb}(c) \text { and }\left(n_{j}\right)_{j \in \mathbb{N}} \text { is such that } x \in \cap_{j} \mathcal{D}_{n_{j}} .
\end{aligned}\right.
$$

In $\mathrm{BHa}$ it was shown how to extend the map $\pi_{\rho} \circ P^{-1}$ to a measurable factor map $\tilde{\pi}: \operatorname{Bas}(\mathcal{A}) \rightarrow \mathbb{S}^{1}$. Here we will give a construction of $\tilde{\pi}$ which is more closely connected to $[\mathrm{BKS}]$.

For any $x$ with $\hat{\chi}_{n}(x) \rightarrow \infty$, the number $b_{n}(x)$ defined as

$$
b_{n}(x):=\max _{j}\left\{j: f^{j}\left(Z_{j}(x)\right)=\mathcal{D}_{n}\right\}
$$

exists. If $x \in f^{-k}(c)$ for some $k \geq 0$, then we need to write $Z_{n}^{ \pm}(x)$ for $n>k$, because $x$ is the common boundary point of two cylinder sets, and $f^{n}(x)=c_{n-k} \in \mathcal{D}_{n-k}$ for $n$ sufficiently large. So $b_{n}(x)$ is well-defined in this case too. Set

$$
\tilde{\pi}_{n}(x):=-\sum_{k} \mathfrak{f p}\left(\rho\left\langle b_{n}(x)-n\right\rangle_{k} S_{k}\right) \bmod 1=-\rho\left(b_{n}(x)-n\right) \bmod 1 .
$$

If $n+1$ is not a cutting time, then $b_{n+1}(x)=b_{n}(x)+1$; in this case $\tilde{\pi}_{n+1}(x)=\tilde{\pi}_{n}(x)$. If $n+1=S_{k}$ is a cutting time, $f^{b_{n}(x)}\left(Z_{b_{n}(x)}(x)\right)=\mathcal{D}_{S_{k}-1}$ and $f^{b_{n}(x)+1}\left(Z_{b_{n}(x)}(x)\right)=\mathcal{D}_{S_{k}}$, but $b_{n+1}(x)$ can be strictly larger than $b_{n}(x)+1$. In this case, however,

$$
b_{n+1}(x)=b_{n}(x)+1+\sum_{j \geq k} d_{j} S_{Q(j)}
$$

for some non-negative integers $d_{j}$. Recall from Lemma 33 that for $\lambda$-a.e. $x \in \operatorname{Bas}(\mathcal{A})$, there is $\hat{k}_{0}=\hat{k}_{0}(x)$ such that for all $k>\hat{k}_{0}$

$$
\text { if } F^{m}(x) \in E_{k} \text {, then } F^{m+j}(x) \notin E_{k} \text { for all } j>C k \text {. }
$$

This means that $\sum_{j \geq k} d_{j} \leq k$, and therefore $\left\langle b_{n}(x)-n\right\rangle$ and $\left\langle b_{n+1}(x)-(n+1)\right\rangle$ are sequences which differ by at most $C k$ entries and the indices of these entries are $\geq k-B$. This means that by the definition of (9)

$$
\left|\tilde{\pi}_{n}(x)-\tilde{\pi}_{n+1}(x)\right| \leq C k \max _{i \geq k-B}\left|\mathfrak{f} \mathfrak{p}\left(\rho S_{i}\right)\right|,
$$

which is summable over $k$ by assumption. It follows that $\left\{\tilde{\pi}_{n}(x)\right\}_{n}$ is a Cauchy sequence in $\mathbb{S}^{1}$ for $\lambda$-a.e. $x \in \operatorname{Bas}(\mathcal{A})$, and hence $\tilde{\pi}(x):=\lim _{n \rightarrow \infty} \tilde{\pi}_{n}(x)$ exists.

Let us complete Step 1 by showing that $\tilde{\pi} \circ f=R_{\rho} \circ \tilde{\pi}$ for $\lambda$-a.e. $x \in \operatorname{Bas}(\mathcal{A})$. Assume that $x \notin \cup_{j} f^{-j}(c)$, then $f\left(Z_{j}(x)\right)=Z_{j-1}(f(x))$ for all sufficiently large $j$. Therefore $b_{n}(f(x))=b_{n}(x)-1$, so substituting into (9) gives $\tilde{\pi}_{n}(f(x))=\tilde{\pi}_{n}(x)+\rho$ for each $n$. In the limit, $\tilde{\pi} \circ f=R_{\rho} \circ \tilde{\pi}$.

Remark 35. It can be shown that $\tilde{\pi}$ is well-defined on $\mathcal{A}$ and coincides with $\pi$, but since it plays no role in Theorem 34, we will omit the proof.

\section{Step 2: The measure of Dis.}

We will show that if $\tilde{\pi}(x) \neq \tilde{\pi}(y)$, then $(x, y)$ form a distal pair. This is more involved than in Proposition 38 below, because $\left.\tilde{\pi}\right|_{\operatorname{Bas}(\mathcal{A})}$ is not continuous. With the exception of a set of measure zero, we can assume that $x$ and $y$ satisfy (10); let $k_{1}=\max \left\{\hat{k}_{0}(x), \hat{k}_{0}(y)\right\}$. Suppose that $\tilde{\pi}(x) \neq \tilde{\pi}(y)$ and take $N \in \mathbb{N}$ and $\eta>0$ such that $\left|\tilde{\pi}_{n}(x)-\tilde{\pi}_{n}(y)\right|>2 \eta$ for 
all $n \geq N$. Take $k_{2} \geq k_{1}$ so large that $C k \max _{i \geq k-B}\left|\mathfrak{f p}\left(\rho S_{i}\right)\right|<\eta$ for all $k \geq k_{2}$ and $C$ as in Lemma 33 ,

We know that $\mathcal{A} \subset \cup_{n \geq S_{k_{2}}} \mathcal{D}_{n}$, but by Proposition 32, part (c), $\omega(c) \subset \cup_{n=1+S_{k_{2}}}^{S_{k_{2}+B}} \mathcal{D}_{n}$. Take $\varepsilon>0$ so small that every two intervals $\mathcal{D}_{n}, \mathcal{D}_{n^{\prime}}, S_{k_{2}}<n, n^{\prime} \leq S_{k_{2}+B}$ either are at least $\varepsilon$ apart or their intersection has length at most $\varepsilon$.

For $\lambda$-a.e. $x \in \operatorname{Bas}(\mathcal{A})$, we have that $\hat{\chi}_{n}(x) \rightarrow \infty$. Hence $f^{i}(x) \in \mathcal{D}$ for $i$ sufficiently large, even though $\mathcal{D}$ only contains a "one-sided" open neighbourhood of $\omega(c)$. (In fact it is possible to prove that the same statement is true for all $x \in \operatorname{Bas}(\mathcal{A})$, but this is enough for our purposes.)

Suppose now by contradiction that $(x, y)$ is not distal. Then there is $i \geq N$ such that $f^{i}(x), f^{i}(y) \in \mathcal{D}$ and $\left|f^{i}(x)-f^{i}(y)\right|<\varepsilon$. So $f^{i}(x)$ and $f^{i}(y)$ belong to the same interval $\mathcal{D}_{n}$ for some $n \geq S_{k_{2}}$, and taking $i$ larger if necessary, we can assume that $n$ is a cutting time. By (10), $\left\langle b_{n}(x)-i\right\rangle$ and $\left\langle b_{n}(y)-i\right\rangle$ are sequences which differ by at most $C k$ entries and the indices of these entries are $\geq k-B$. Similar to (11), we have

$$
\left|\tilde{\pi}_{n}(x)-\tilde{\pi}_{n}(y)\right| \leq C k \max \left\{\left|\mathfrak{f} \mathfrak{p}\left(\rho S_{i}\right)\right|: k-B \leq i \leq 2 k-B\right\}<\eta .
$$

This contradiction to the choice of $\eta$ and $N$ proves that $(x, y)$ is distal. Therefore proximal pairs $(x, y)$ can only exist within fibers of $\tilde{\pi}$.

Finally, if $W=\tilde{\pi}^{-1}(s)$ with $\lambda(W)>0$ for some $s \in \mathbb{S}^{1}$, then since $\tilde{\pi} \circ f=R_{\rho} \circ \tilde{\pi}$ and $R_{\rho}$ is invertible, it follows that $f^{m}(W) \cap f^{n}(W)=\emptyset$ for all $0 \leq m<n$, and this contradicts the non-existence of strongly wandering sets. Therefore each fiber has measure zero. This completes the proof.

Corollary 36. If $S_{k}=S_{k-1}+S_{k-d}$ for $d=2,3,4$, and $f$ is a map with cutting times $\left\{S_{k}\right\}_{k \geq 0}$ and sufficiently large critical order so that $\mathcal{A}$ is a wild attractor with positive drift, then $\lambda_{2}$ (Dis) $=1$.

Proof. We know from [BKS] that the dynamics $(\mathcal{A}, f)$ is semi-conjugate to a minimal rotation on a $d$-1-dimensional torus. If fact, the characteristic equation $\lambda^{d}=\lambda^{d-1}+1$ of the recursive relation $S_{k}=S_{k-1}+S_{k-d}$ has a leading root $\rho$ which is a Pisot number, i.e., all its algebraic conjugates lie within the unit disk. It follows easily that $\mathfrak{f p}\left(\rho S_{k}\right)$ is exponentially small in $k$ so that the condition $\sum_{k} k\left|\mathfrak{f p}\left(\rho S_{k}\right)\right|<\infty$ is obviously satisfied, and Theorem 34 shows that $\lambda_{2}(\mathrm{Dis})=1$. By defining $\Pi: \operatorname{Bas}(\mathcal{A}) \rightarrow \mathbb{T}^{d-1}$ as $\Pi(x)=\left(\tilde{\pi}_{\rho}(x), \ldots, \tilde{\pi}_{\rho^{d-1}}(x)\right)$ (which is well-defined $\lambda$-a.e.), we obtain a factor map onto $\left(\mathbb{T}^{d-1}, R_{\rho, \ldots, \rho^{d-1}}\right)$ with Haar measure, which is the maximal automorphic factor of $(\operatorname{Bas}(\mathcal{A}), \lambda, f)$.

Proposition 37. Under the conditions of Theorem 34 and if there is a continuous factor of $(\mathcal{A}, f)$ onto a d-dimensional torus $\mathbb{T}^{d}$ (with $\left.d \geq 1\right)$, then the fiber $\tilde{\pi}^{-1}(\tau) \subset \operatorname{Bas}(\mathcal{A})$ for each $\tau \in \mathbb{T}^{d}$ contains an uncountable $\varepsilon$-scrambled set for some $\varepsilon>0$.

Proof. Assume for simplicity that $d=1$ and take $\rho \in \mathbb{R}$ such that $\tilde{\pi} \circ f=\tilde{\pi}+\rho \bmod 1$ for $\lambda$-e.a. $x \in \mathcal{A}$. Because a zero-dimension set cannot be mapped injectively onto a set of higher dimension, see [E], the continuity of the factor map $\pi: \mathcal{A} \rightarrow \mathbb{T}^{d}$ implies that $\pi$ cannot be injective. Therefore we can find $a \neq \hat{a} \in \mathcal{A}$ such that $f(a)=f(\hat{a})$. By Proposition 32, part (c), we can find, for every $k$, integers $\kappa$ and $\hat{\kappa}$ with $k<\kappa, \hat{\kappa} \leq k+B$ and $N \leq \min \left(S_{\kappa}, S_{\hat{\kappa}}\right)$, such that $\mathcal{D}_{S_{\kappa}-N} \ni a$ and $\mathcal{D}_{S_{\hat{\kappa}}-N} \ni \hat{a}$. 
Hence we can find two sequences $\left(\kappa_{j}\right)_{j \in \mathbb{N}}$ and $\left(\hat{\kappa}_{j}\right)_{j \in \mathbb{N}}$ with $\left|\kappa_{j}-\hat{\kappa}_{j}\right| \leq B$, but possibly $\kappa_{j+1} \gg \kappa_{j}$, and another sequence $\left(N_{j}\right)_{j \in \mathbb{N}}$ such that $\mathcal{D}_{S_{\kappa_{j}}-N_{j}} \ni a$ and $\mathcal{D}_{S_{\hat{\kappa}_{j}}-N_{j}} \ni \hat{a}$.

Recall the Markov map was defined as $F: \sqcup_{l \geq 0} E_{l} \rightarrow \sqcup_{l \geq 0} E_{l}$. For each $j$, we will create loops from $E_{\kappa_{j}}$ to itself and from $E_{\hat{\kappa}_{j}}$ to itself, as indicated in Figure 2. Both loops require the same steps under $F$, only arranged in a different order, hence they involve the same number $s_{j}$ of iterates of $f$. Because $\lim \sup _{k} k-Q(k) \leq B$, both loops involve no more than $2 B$ steps, and the width (highest vertex minus smallest vertex) is less than $2 B$ as well.
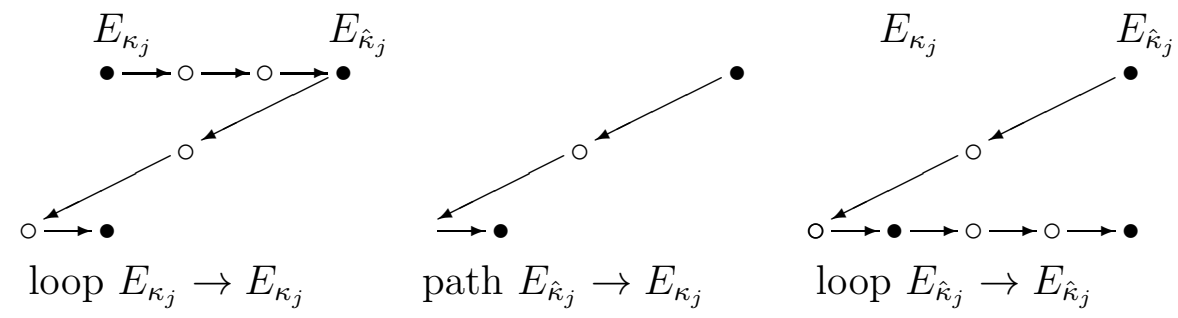

Figure 2. Different loops ending at $E_{\kappa_{j}}$ and $E_{\hat{\kappa}_{j}}$ respectively. Both loops contain the same path from $E_{\hat{\kappa}_{j}}$ to $E_{\kappa_{j}}$, depicted in the middle. Backward arrows go from $E_{l}$ to $E_{Q(l+1)}$.

Assume that $\kappa_{j}<\hat{\kappa}_{j}$ and $E_{\kappa_{j}} \supset E_{\hat{\kappa}_{j}}$. (The other three cases can be treated similarly.) Since $\left\{E_{l}\right\}_{l \geq 0}$ is a Markov partition for $F$, there are intervals $J_{j} \subset E_{\kappa_{j}}$ and $\hat{J}_{j} \subset E_{\hat{\kappa}_{j}}$ such that $f^{s_{j}}: J_{j} \rightarrow E_{\kappa_{j}}$ and $f^{s_{j}}: \hat{J}_{j} \rightarrow E_{\hat{\kappa}_{j}}$ are diffeomorphic and onto. There is a similar interval $\hat{K}_{j} \subset E_{\hat{\kappa}_{j}}$ representing the path from $E_{\hat{\kappa}_{j}}$ to $E_{\kappa_{j}}$, that is if the path from $E_{\hat{\kappa}_{j}}$ to $E_{\kappa_{j}}$ requires $t_{j}$ iterates of $f$, then $f^{t_{j}}: \hat{K}_{j} \rightarrow E_{\kappa_{j}}$ is diffeomorphic and onto.

Combining the two, we find intervals $H_{j} \subset J_{j}$ such that

$$
\left\{\begin{array}{l}
\text { (a) } f^{s_{j}}\left(H_{j}\right)=\hat{K}_{j} \subset E_{\hat{\kappa}_{j}} \subset E_{\kappa_{j}}, \\
(b) f^{s_{j}+t_{j}}\left(H_{j}\right)=E_{\kappa_{j}} \text {, and } \\
(c) f^{s_{j}-\left(N_{j}+1\right)}\left(H_{j}\right) \text { contains, or is close to, } a .
\end{array}\right.
$$

Similarly, we can find $\hat{H}_{j} \subset \hat{J}_{j}$ such that

$$
\left\{\begin{array}{l}
(\hat{a}) \quad f^{s_{j}}\left(\hat{H}_{j}\right)=\hat{K}_{j} \subset E_{\hat{\kappa}_{j}}, \\
(\hat{b}) \quad f^{s_{j}+t_{j}}\left(\hat{H}_{j}\right)=E_{\kappa_{j}}, \text { and } \\
(\hat{c}) \quad f^{s_{j}-\left(N_{j}+1\right)}\left(\hat{H}_{j}\right) \text { contains, or is close to, } \hat{a} .
\end{array}\right.
$$

Let $\Sigma \subset\{0,1\}^{\mathbb{N}}$ be an uncountable scrambled subset of the full shift. The idea is now, for each $\tau \in \mathbb{T}$ and each $\sigma \in \Sigma$, to find a point $x \in \pi^{-1}(\tau)$ such that

$$
f^{r_{j}}(x) \in \begin{cases}\mathcal{D}_{S_{\kappa_{j}}-N_{j}} \ni a & \text { if } \sigma_{j}=0, \\ \mathcal{D}_{S_{\hat{\kappa}_{j}}-N_{j}} \ni \hat{a} & \text { if } \sigma_{j}=1,\end{cases}
$$

where the sequence $r_{j}$ depends of $t$ but not on $\sigma$.

Start with some $y \in \mathcal{D}_{2}=E_{0}$ with $\tilde{\pi}(y)=\tau+\varepsilon$ where $\varepsilon$ will be determined later. Then, when the orbit of $y$ under iteration of $F$ goes from $E_{\kappa_{j}}$ to $E_{\hat{\kappa}_{j}}$, we insert one of the loops as in Figure 2 according to whether $\sigma_{j}=0$ or 1 , and the extra path from $E_{\kappa_{j}}$ to $E_{\hat{\kappa}_{j}}$. 
That is, when $q_{1}$ is such that $f^{q_{1}}(y) \in E_{\kappa_{j}}$, we insert one of the extended loops, both taking $s_{1}+t_{1}$ iterates, and iterate $r_{1}:=q_{1}+s_{1}-\left(N_{1}+1\right)$ brings the path close to $a$ or $\hat{a}$, depending on whether $\sigma_{1}=0$ or 1 . Then, when $y$ visit $E_{\kappa_{2}}$, the new extended loop takes $s_{1}+t_{1}$ iterates more to reach it; call this number $q_{2}$, insert the appropriate extended loop of $s_{2}+t_{2}$ iterates, and find that after $r_{2}=q_{2}+s_{2}-\left(N_{2}+1\right)$ iterates, the path will be close to $a$ or $\hat{a}$, etc.

Due to the Markov property, there is some $x \in E_{0}$ whose infinite path under $F$ is precisely the path we have created, so $x$ satisfies (12). Furthermore, the values $b_{n}(x)=b_{n}(y)+$ $\sum_{j, q_{j}+B<n} s_{j}+t_{j}$. When we compute $\tilde{\pi}(x)$, the contribution $\varepsilon$ of all the inserted extended loops bounded by

$$
\sum_{j} 3 B \max _{\kappa_{j}-B \leq i \leq \kappa_{j}+B}\left|\mathfrak{f p}\left(\rho S_{i}\right)\right|<\infty .
$$

Here we used that each loop requires at most $2 B$ steps and each path from $E_{\kappa_{j}}$ to $E_{\hat{\kappa}_{j}}$ (or vice versa) has at most $B$ steps, and that all these steps are taken within a distance $B$ from the step $E_{\kappa_{j}} \rightarrow E_{\kappa_{j}+1}$.

In particular, $\tilde{\pi}(x)$ is well-defined, and $\tilde{\pi}(x)=\tilde{\pi}(y)-(\tilde{\pi}(y)-\pi(x))=\tilde{\pi}(y)-\varepsilon=\tau$, so $x \in \tilde{\pi}^{-1}(\tau)$.

Finally, if $\sigma \neq \sigma^{\prime}$ both belong to $\Sigma$, there are infinitely many $j$ s such that $\sigma_{j} \neq \sigma_{j}^{\prime}$, and consequently $\limsup _{j \rightarrow \infty}\left|f^{r_{j}}(x)-f^{r_{j}}\left(x^{\prime}\right)\right|=|a-\hat{a}|>0$. On the other hand, $\lim _{j \rightarrow \infty}\left|f^{q_{j}}(x)-f^{q_{j}}\left(x^{\prime}\right)\right|=0$, because $f^{q_{j}}(x)$ and $f^{q_{j}}\left(x^{\prime}\right)$ both belong to $E_{\kappa_{j}}$ or to $E_{\hat{\kappa}_{j}}$ Therefore $\{x(\sigma): \sigma \in \Sigma\}$ is an $|a-\hat{a}|$-scrambled subset of $\pi^{-1}(\tau)$, as required.

Proposition 38. Let $f$ be a unimodal map with kneading map $Q(k)=\max \{k-d, 0\}$ for $d=2,3,4$, and let $\mu$ denote the unique invariant probability measure supported by $\omega(c)$. Then

- $\mu_{2}$-a.e. pair of points is distal;

- if $d=2$ (the Fibonacci map) then $\omega(c)$ contains no Li-Yorke pair, and the only asymptotic pairs $(x, y)$ are such that $f^{n}(x)=f^{n}(y)=c$ (only one such pair for each $n \geq 1)$

- if $d=3,4$ then there are uncountably many Li-Yorke pairs in $\omega(c)$.

Proof. As in Corollary [36, there is a continuous map $\pi: \omega(c) \rightarrow \mathbb{T}^{d-1}$ onto the $d-1$ dimensional torus and an irrational rotation $R:=R_{\rho, \ldots, \rho^{d}}: \mathbb{T}^{d} \rightarrow \mathbb{T}^{d}$ such that $\pi \circ f=R \circ \pi$. If $\pi(x) \neq \pi(y)$ then $(x, y)$ is distal, as in Corollary [36. It follows from [BKS] that this happens for $\mu_{2}$-a.e. $(x, y)$.

Furthermore, $\pi^{-1}(b)$ consists of at most $d$ points $a_{1}, \ldots, a_{d}$ for any $b \in \mathbb{T}^{d-1}$. If there are indeed $d$ distinct points, then there is $n \geq 0$ such that $f^{n}\left(a_{1}\right)=f^{n}\left(a_{2}\right)=\cdots=f^{n}\left(a_{d}\right)=c$, cf. [Br4]. For the Fibonacci map, this accounts for all non-distal pairs. For $d=3$, 4, other non-singleton fibers $\pi^{-1}(b)$ are possible, and Li-Yorke pairs exists within such fibers. They are related to incidences in the substitution shift description, as described implicitly in $[\mathrm{BD}]$.

The question is whether the situation is the same for the "next" Fibonacci-like map with $Q(k)=\max \{k-5,0\}$. In this case, the system of $(\omega(c), f)$ with its unique probability 
measure is weak mixing, so there is no continuous (or even measurable) factor map onto a group rotation. The difference with the previous cases is that the characteristic equation of the recursive relation

$$
0=\lambda^{5}-\lambda^{4}-1=\left(\lambda^{2}-\lambda+1\right)\left(\lambda^{3}-\lambda-1\right)
$$

is reducible, and more decisively, its leading root is not a Pisot number. The following curiosity about the cutting times holds in this case:

$$
S_{k}=S_{k-2}+S_{k-3}+\left\{\begin{aligned}
+1 & \text { if } k \equiv 2,3 \bmod 6 \\
-1 & \text { if } k \equiv 5,0 \bmod 6 \\
0 & \text { if } k \equiv 1,4 \bmod 6
\end{aligned}\right.
$$

Note that the same algebraic curiosity holds for any characteristic equation $\lambda^{6 m-1}-$ $\lambda^{6 m-2}-1=0$, because in each such case, $\lambda^{2}-\lambda+1$ (with solutions $\lambda=\frac{1 \pm i \sqrt{3}}{2}$ on the unit circle) divides the equation. As an example, the case $m=2$ gives:

$$
\lambda^{11}-\lambda^{10}-1=\left(\lambda^{2}-\lambda+1\right)\left(\lambda^{9}-\lambda^{7}-\lambda^{6}+\lambda^{4}+\lambda^{3}-\lambda-1\right),
$$

and one can indeed check that

$$
S_{k}=S_{k-2}+S_{k-3}-S_{k-5}-S_{k-6}+S_{k-8}+S_{k-9}+\left\{\begin{aligned}
+1 & \text { if } k \equiv 2,3 \bmod 6 \\
-1 & \text { if } k \equiv 5,0 \bmod 6 \\
0 & \text { if } k \equiv 1,4 \bmod 6
\end{aligned}\right.
$$

Proposition 39. If $S_{k}=S_{k-1}+S_{Q(k)}$ for $Q(k)=\max \{0, k-5\}$ and $f$ is a map with cutting times $\left\{S_{k}\right\}_{k \geq 0}$ and a wild attractor with positive drift, then $\lambda_{2}\left(\mathrm{LY}_{\varepsilon}\right)=1$ for some $\varepsilon>0$.

Proof. As pointed out before, it was shown in [Br3] that $\mathcal{A}=\omega(c)$ is a wild attractor, and from [BHa] it follows that dynamics on the basin of the attractor is Lebesgue exact. Thus Proposition 27 implies that $\lambda_{2}(\mathrm{LY})=1$. In fact, the construction of Proposition $37 \mathrm{can}$ also be used here to show that there is $\varepsilon>0$ such that $\lambda_{2}$-a.e. pair belongs to $L Y_{\varepsilon}$.

Example 40. Let $f$ be a unimodal map with cutting times satisfying

$$
S_{0}=1, S_{1}=2, S_{2}=3, S_{3}=4, S_{4}=6, S_{5}=8, S_{6}=10, S_{7}=12
$$

and

$$
S_{k}=S_{k-1}+S_{k-5} \text { for } k \geq 8 \text {. }
$$

This means that the cutting times $S_{k}$ are even for $k \geq 3$ and eventually are twice the numbers occurring in the example of Proposition [39. Assume also that the critical order of $\ell$ is so large that $f$ has a wild attractor $\mathcal{A}$. Then $\mathcal{A}$ decomposes into two disjoint Cantor sets $\mathcal{A}_{0}$ and $\mathcal{A}_{1}$ which are permuted by $f$. Note, however, that $f$ is not renormalizable, see Proposition 32, and therefore $f$ is topologically mixing on $\left[c_{2}, c_{1}\right]$.

Let $B_{0}$ and $B_{1}$ be disjoint neighborhoods of $\mathcal{A}_{0}$ and $\mathcal{A}_{1}$; for example we can take $B_{0}=$ $\left[c_{2}, c_{14}\right] \cup\left[c_{4}, c_{6}\right]$ and $B_{1}=\left[c_{3}, c_{15}\right] \cup\left[c_{5}, c_{1}\right]$. Every point in the basin of $\mathcal{A}$ will eventually be trapped in $B_{0} \cup B_{1}$. But every pair $(x, y)$ with $x \in B_{0}$ and $y \in B_{1}$ such that $\operatorname{orb}(x) \operatorname{orb}(y) \subset B_{0} \cup B_{1}$ is distal. On the other hand $\left.f^{2}\right|_{\mathcal{A}_{0}}$ and $\left.f^{2}\right|_{\mathcal{A}_{1}}$ behave like the example of Proposition [39, so $\lambda_{2}$-a.e. every pair $(x, y) \in B_{0} \times B_{0} \quad\left(\right.$ or $\left.(x, y) \in B_{1} \times B_{1}\right)$ such that $\operatorname{orb}(x), \operatorname{orb}(y) \subset B_{0} \cup B_{1}$ is Li-Yorke. 
Proof of Theorem E. From Theorem C we know that $\lambda_{2}($ Prox $)=0$. Also, if some $x \in \operatorname{Bas}(\mathcal{A})$ is approximately periodic, then by Proposition $[31$, $\mathcal{A}$ is conjugate to an adding machine, so all points in $\operatorname{Bas}(\mathcal{A})$ are approximately periodic. By Proposition 6 , $\operatorname{Bas}(\mathcal{A})$ contains no Li-Yorke pairs. Therefore $(\mathrm{a})-(\mathrm{d})$ are the only possibilities, and they all occur:

(a) The strange adding machine case as wild attractor, see [Br4].

(b) The Fibonacci-like map with kneading map $Q(k)=\max \{k-d, 0\}, d=2,3$, 4, see Theorem 34 and Corollary 36.

(c) The Fibonacci-like map with kneading map $Q(k)=\max \{k-5,0\}$, see Proposition 39 ,

(d) See Example 40.

Proposition 37 implies the existence of $\varepsilon$-scrambled sets in the fibers $\tilde{\pi}^{-1}(\tau)$ for all $\tau \in \mathbb{S}^{1}$ and factor maps $\tilde{\pi}$ in case (b), and for cases (c) and (d), the $\varepsilon$-scrambled set is also immediate. Li-Yorke sensitivity follows as well.

Remark 41. In case (a) and (b) of Theorem $E,\left(\mathcal{A}, \mu_{\mathcal{A}}, f\right)$ is not weakly mixing. Instead, there is an $f_{2}$-invariant set of positive $\mu_{2}$-measure that is bounded away from the diagonal of $\mathcal{A}_{2}$. We expect that case (c) always corresponds to the weakly mixing case, cf. Proposition 39, and in particular, $\mu_{\mathcal{A}} \times \mu_{\mathcal{A}}$-a.e. pair $(x, y)$ has a dense orbit in $\mathcal{A} \times \mathcal{A}$, and hence is Li-Yorke.

Remark 42. In case (a), points $x$ in the basin of $\mathcal{A}$ have a distinct target point $t_{x} \in \mathcal{A}$ such that $\operatorname{dist}\left(f^{n}(x), f^{n}\left(t_{x}\right)\right) \rightarrow 0$, see Proposition [6. In case (b), such target points $t_{x} \in \mathcal{A}$ do not exist in general, cf. the proof of Proposition 37 and also [BHa, Remark 2].

\section{Appendix}

In this appendix, we prove the more technical results of the paper, divided into four parts. First we give a result on the structure of solenoidal sets of Feigenbaum type, next we prove Theorem 20, then we formulate an improved $C^{3}$ Koebe distortion lemma, and finally we prove Theorem 21,

7.1. Neigborhoods of Solenoidal Sets. Assume that $S$ is a solenoidal set and cyc $(K)$ is a solenoidal cycle of period $r$ containing $S$. Let $S_{i}=S \cap f^{i}(K), 0 \leq i<r$. Since the critical points in cyc $(K)$ belong to $S$, the convex hulls $J_{i}$ of $S_{i}$ form a cycle of intervals, that is, $f\left(J_{i}\right)=J_{i+1}$ for every $i$ (with this we also mean $\left.f\left(J_{r-1}\right)=J_{0}\right)$. We call it the $r$-minimal solenoidal cycle covering $S$. We emphasize that the intervals $J_{i}$ are pairwise disjoint (because a solenoid contains no periodic points). Moreover, if they are ordered in the real line as $J_{i_{1}}<J_{i_{2}}<\cdots<J_{i_{r}}$, then there is a union of periodic orbits $P=\left\{p_{i}\right\}_{i=1}^{r-1}$ such that $J_{i_{1}}<p_{1}<J_{i_{2}}<p_{2}<\cdots<p_{r-1}<J_{i_{r}}$ (see [MT] or [AJS]).

The existence of these periodic orbits of smaller period interlaced among the intervals $J_{i}$ allows us to prove immediately that the union $L_{i}$ of all periodic intervals of period $r$ containing $J_{i}$ is also periodic of period $r$. Clearly, $f\left(L_{i}\right) \subset L_{i+1}$ and $f\left(\partial L_{i}\right) \subset \partial L_{i+1}$ for every $i$. However, although the intervals $L_{i}$ have pairwise disjoint interiors, they do not form a cycle of intervals because $f\left(L_{i}\right)=L_{i+1}$ need not hold. In fact $\left(L_{i}\right)_{i=0}^{r}$ is the pullback chain of $L_{r}:=L_{0}$ along $J_{0}, J_{1}, \ldots, J_{r}=J_{0}$. Let $M_{i}=\operatorname{Int} L_{i}$. We call $M=\bigcup_{i=0}^{r-1} M_{i}$ the $r$-shell covering $S$. Then $M$ is nice and $J_{i} \subset M_{i}$ for every $i$. 
Proposition 43. Let $f \in C_{\mathrm{nf}}^{2}(I)$ and let $S$ be a solenoidal set of Feigenbaum type. Let $T$ be the $r$-minimal solenoidal cycle covering $S$ for some $r, T=\bigcup_{i=0}^{r-1} f^{i}(J)$, and let $M=\bigcup_{i=0}^{r-1} M_{i}$ be the $r$-shell covering $S$, with $f^{i}(J) \subset M_{i}$ for every $0 \leq i<r$. Then there is $\xi=\xi(f)>0$ such that $f^{i}(J)$ is $\xi$-well centered in $M_{i}$ for every $i$.

Proof. Since $S$ is a solenoid, there is a turning point $c \in S$. There is no loss of generality in assuming that $c \in J$. Also, we can assume that $r$ is large enough so that each interval $M_{i}$ contains at most one critical point of $f$ and there are no critical points in $\mathrm{Cl} M$ outside $T$. Hence $\left(M_{i}\right)_{i=0}^{r}$ is the pullback chain of $M_{r}:=M_{0}$ along $J, f(J), \ldots, f^{r}(J)=J$. Let cyc $(R)$ be the $2 r$-minimal solenoidal cycle covering $S$, with $c \in R$. We can for example assume that $[u, v]=R, f^{r}(R)=[w, z]$ and $c \in R$ is a local maximum, Note that $J$ is the convex hull of $R \cup f^{r}(R)$. Observe that there is a periodic point $p$ of period $r$ such that $f^{i}(p)$ lies between $f^{i}(R)$ and $f^{i+r}(R)$ for every $i$. Since $T$ is a solenoidal cycle, orb $(p)$ is hyperbolic repelling. In fact, since $f$ is monotone on each on the intervals connecting $f^{i}(R)$ and $f^{i+r}(R)$, it is the only $r$-periodic orbit in $T$ and $f^{r}([u, p))=(p, z], f^{r}((p, z])=[u, p)$. Then $\left(\tau_{c}(p), p\right)$ is clearly the only nice periodic interval of period $2 r$ containing $c$.

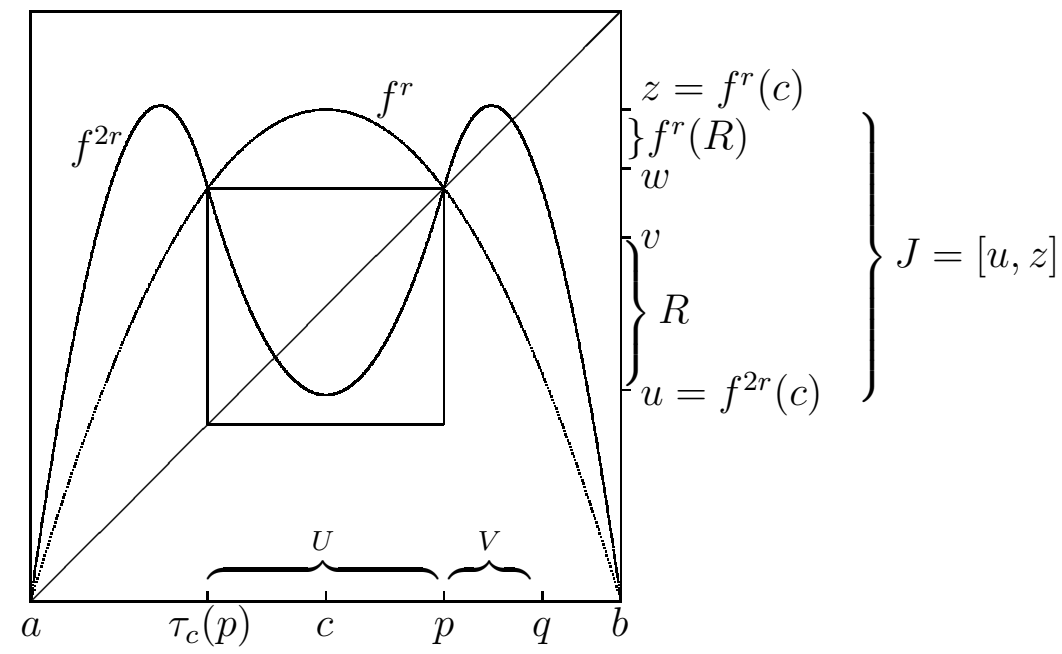

FiguRE 3. The graph of $f^{r}: M_{0}=(a, b) \rightarrow M_{0}$ with $J$ and $R$ and relevant points

By $\left[\mathrm{SV}\right.$, Theorem $\left.\mathrm{A}^{\prime}(1)\right]$ there are an integer $s>0$, a number $\xi_{0}=\xi_{0}(f)>0$ and nice periodic intervals $N^{m} \ni c$ of periods $s 2^{m}, m \geq 0$, such that $N^{m+1}$ is $\xi_{0}$-well inside $N^{m}$ for every $m$. We have shown in the previous paragraph that if $s 2^{m}$ is large enough, then there is just one nice periodic interval of period $s 2^{m}$ containing $c$. Thus we can assume that $M_{0}=N^{m}$ and $\left(\tau_{c}(p), p\right)=N^{m+1}$ for some $m$, with $r=s 2^{m}$.

Let $q>p$ be the closest point to $p$ satisfying $f^{r}(q)=\tau_{c}(p)$. Then $V=(p, q)$ is just the pullback of $U=\left(\tau_{c}(p), p\right)$ along $f^{r}(R), f^{r+1}(R), \ldots, f^{2 r}(R)=R$. Since $U$ is $\xi_{0^{-}}$well inside $M_{0}$, there is $\xi_{1}=\xi_{1}(f)$ such that $V$ is $\xi_{1}$-well inside $M_{0}$, due to Corollary 14. 
Let $W=(q, b]$ with $b$ the right endpoint of $M_{0}$. Then $\xi_{0}|U| \leq|V|+|W|$ and $\xi_{1}|V| \leq|W|$, hence

$$
\frac{\xi_{0} \xi_{1}}{1+\xi_{0}+\xi_{1}}(|U|+|V|) \leq|W|
$$

Then $\left(\tau_{c}(q), q\right)$ is well centered in $M_{0}$ and so is any subinterval of $\left(\tau_{c}(q), q\right)$. In particular, $J$ is well centered in $M_{0}$.

To finish the proof we must show that every interval $f^{i}(J)$ is well centered in the corresponding interval $M_{i}$. Note that we cannot directly use Corollary 14 because it only guarantees that $f^{i}(J)$ is well inside $M_{i}$. Instead we proceed as follows. According to [SV, Lemma 2], $M_{r}:=M_{0}$ is well inside an interval $G_{r}$ which contains at most $e=2^{d+1}+3$ of the intervals $f^{i}\left(M_{0}\right), 0 \leq i<r$, with $d=$ \# Crit. Therefore it also contains at most $e$ of the intervals $M_{i}$. Now [SV, Lemma 3] implies that the pullback chain $\left(G_{i}\right)_{i=0}^{r}$ of $G_{r}$ along $M_{0}, \ldots, f^{r}\left(M_{0}\right)$ has order bounded by $2(e+d(e+2))+1$. Hence, by Corollary 14, $M_{i}$ is well inside $G_{i}$ for every $i$ and, additionally, if some iterate $f^{l}$ maps diffeomorphically $M_{i}$ onto $M_{i+l}$, then this diffeomorphism has bounded distortion. Thus, if $f^{i+l}(J)$ is well centered in $M_{i+l}, f^{i}(J)$ is well centered in $M_{i}$. Using now Lemma 11 and recalling that $J=f^{r}(J)$ is well centered in $M_{0}=M_{r}$, we conclude that every interval $f^{i}(J)$ is well centered in $M_{i}$ as we desired to show.

7.2. Proof of Theorem 20. Later on we will apply the lemma below to the set $Q=$ Crit of critical points of our map $f \in C_{\mathrm{nf}}^{2}(I)$; recall that we are assuming that $f$ has no periodic critical points, see (2).

Lemma 44. Let $f: I \rightarrow I$ be a multimodal map without wandering intervals and let $Q \subset I \backslash \partial I$ be a finite set containing no periodic points. Let $x \in Q$. Then there is an arbitrarily small nice interval $J \ni x$ such that $\operatorname{orb}(Q) \cap \partial J=\emptyset$ and $\operatorname{dist}(\operatorname{orb}(\partial J), Q)>0$.

Proof. Let $Q^{\prime}=\bigcup_{n=-\infty}^{\infty} f^{n}(Q)$. We claim that the set $P=\operatorname{AsPer} \backslash Q^{\prime}$ is dense in $I$. This implies the lemma. Indeed, let $\varepsilon>0$ be small. Since $x$ is not periodic, there is no loss of generality in assuming that $\operatorname{orb}(Q) \cap(x-\varepsilon, x+\varepsilon)$ does not contain any periodic point. Take points $\hat{a} \in(x-\varepsilon, x) \cap P, \hat{b} \in(x, x+\varepsilon) \cap P$, and let $a<x<$ $b$ be the points from $\mathrm{Cl}(\operatorname{orb}(\hat{a}) \cup \operatorname{orb}(\hat{b}))$ closest to $x$ from both sides. We emphasize that both $\hat{a}$ and $\hat{b}$ are asymptotically periodic and $x$ is not periodic, so $a$ and $b$ are well defined. Moreover, $(a, b)$ is nice. Also, $a \notin Q^{\prime}$. If $a \in \operatorname{orb}(\hat{a}) \cup \operatorname{orb}(\hat{b})$ this is obvious because $\hat{a}, \hat{b} \notin Q^{\prime}$. If $a \notin \operatorname{orb}(\hat{a}) \cup \operatorname{orb}(\hat{b})$, then $a$ must belong to a periodic orbit attracting either $\operatorname{orb}(\hat{a})$ or orb $(\hat{b})$, and again $a \notin Q^{\prime}$ because $Q$ contains no periodic points and neither does $\operatorname{orb}(Q) \cap(x-\varepsilon, x+\varepsilon)$. Similarly, $b \notin Q^{\prime}$. We have shown $\operatorname{orb}(Q) \cap\{a, b\}=\emptyset$ and $(\operatorname{orb}(a) \cup \operatorname{orb}(b)) \cap Q=\emptyset$. Since $a$ and $b$ are asymptotically periodic and $Q$ contains no periodic points, the property $(\operatorname{orb}(a) \cup \operatorname{orb}(b)) \cap Q=\emptyset$ implies in fact $\operatorname{dist}(\operatorname{orb}(a) \cup \operatorname{orb}(b), Q)>0$. Thus $J=(a, b)$ is the small nice interval we are looking for.

We prove that every interval $K$ intersects $P$. If the intervals $\left\{f^{n}(K)\right\}_{n}$ are pairwise disjoint, then the absence of wandering intervals for $f$ implies that these intervals are attracted by a periodic orbit. Since $f$ is multimodal, the set of points in $K \cap Q^{\prime}$ is countable. Thus $K$ intersects $P$. 
Now assume that $f^{n}(K) \cap f^{m}(K) \neq \emptyset$ for some $n<m$. Let $k=m-n$. Using again that $f$ is multimodal, we get that $T=\mathrm{Cl}\left(\bigcup_{r=0}^{\infty} f^{n+r k}(K)\right)$ is a nondegenerate interval. Moreover, it is invariant for $f^{k}$. If $\left.f^{k}\right|_{T}$ has finitely many periodic points, then all points from $T$ are asymptotically (or eventually) periodic, see e.g. [BC, p. 127], so all points from $K$ are asymptotically periodic as well and $K \cap P \neq \emptyset$ as before. If $\left.f^{k}\right|_{T}$ has infinitely many periodic points, there is a family of disjoint periodic orbits $\left\{\mathcal{O}_{j}\right\}_{j=1}^{\infty}$ such that $\operatorname{orb}(K) \cap \mathcal{O}_{j} \neq \emptyset$ for every $j$. If $j$ is large enough, then $\operatorname{orb}(Q) \cap \mathcal{O}_{j}=\emptyset$. Let $y \in K$ be a preimage of such $\mathcal{O}_{j}$. Then $y \notin$ fullorb $(Q)$, which finishes the proof.

The next proposition strengthens [CL, Proposition 5]. In what follows, we say that a nice set $V$ is $\xi$-nice if all return domains to $V$ are $\xi$-well inside the components of $V$ containing them. Sometimes we say that $V$ is uniformly nice if it is $\xi$-nice for some constant $\xi$ depending only on $f$. We denote

$$
Z=\{x \in I: x \notin \operatorname{orb}(\text { Crit }), \operatorname{dist}(\operatorname{orb}(x), \text { Crit })>0\} .
$$

Observe that $f^{-1}(Z)=Z$ and that (reasoning as in the proof of Lemma 44) $Z$ is dense for $f \in C_{\mathrm{nf}}^{2}(I)$. We denote by Feig the critical points of Feigenbaum type.

Proposition 45. Let $f \in C_{\mathrm{nf}}^{2}(I)$. Then there are $\xi_{0}=\xi_{0}(f)>0$ and, for every $\varepsilon>0$ and $c \in$ Crit $\backslash$ Feig, open intervals $c \in V_{c}$ with $\left|V_{c}\right|<\varepsilon$, such that $V=\bigcup_{c \in \text { Crit } \backslash \text { Feig }} V_{c}$ is $\xi_{0}$-nice and $\partial V \subset Z$.

Proof. In Cai and Li's version of this proposition, all critical points of solenoidal type (not only those of Feigenbaum type) are excluded and no additional properties on $\partial V$ are obtained. Nevertheless, the proof remains very much the same. We sketch it below, emphasizing the specific points where it must be modified.

First of all, we prove:

Claim 1. There is $\xi_{1}=\xi_{1}(f)$ such that if $c \in$ Crit $\backslash$ Feig, then there are arbitrarily small $\xi_{1}$-nice intervals $J$ containing $c$ such that $\partial J \subset Z$.

This is [CL, Corollary 3], but including also solenoidal critical points of non-Feigenbaum type. If $c$ is recurrent, then Claim 1 follows immediately from Propositions 15 and 16. In fact, if $c$ is not solenoidal, and we take as the starting interval $I_{0}$ in Proposition 16 a small nice interval containing $c$ with $\partial I_{0} \subset Z$ (which is possible by Lemma 44), then the central return domain $I_{m}$ to $I_{m-1}$ satisfies $\partial I_{m} \subset Z$ as well for all $m$. Thus if $m$ is large enough and $I_{m+1}$ is well inside $I_{m}$, then, by Proposition 15, $I_{m+1}$ is the uniformly nice interval we need. If $c$ is solenoidal, then Propositions 15] and 16] again imply that there is an arbitrarily small uniformly nice interval $J$ containing $c$. Observe that if $J$ is sufficiently small, then it is contained in a solenoidal cycle very close to $\omega(c)$, which in particular implies that the orbits of its endpoints cannot accumulate on any critical point outside $\omega(c)$. Also, since $J$ is nice and $\omega(c)$ is minimal, they cannot accumulate on $\omega(c)$ either. Thus $\partial J \subset Z$.

If $c$ is not recurrent then the argument from [CL, Corollary 3] applies without any significant changes. Namely, let $I^{\prime} \ni c$ be a small nice interval with $\partial I^{\prime} \subset Z$. We can assume that $c \notin D\left(I^{\prime}\right)$. Let $\delta$ be the minimal length of the components of $I^{\prime} \backslash\{c\}$ and take an interval $\left(a^{\prime}, b^{\prime}\right) \subset(c-\delta / 2, c+\delta / 2)$ with $a^{\prime}, b^{\prime} \in Z$. This is possible by Lemma 44. If 
$a^{\prime} \notin D\left(I^{\prime}\right)$, define $a=a^{\prime}$. Otherwise, let $K$ be the return domain to $I^{\prime}$ containing $a^{\prime}$ and let $a$ be the endpoint of $K^{\prime}$ in $\left(a^{\prime}, c\right)$. The point $b$ is defined similarly. Then $J=(a, b)$ is $1 / 2$-well inside $I^{\prime}, \partial J \subset Z$ and $\partial J \cap D\left(I^{\prime}\right)=\emptyset$. If $x \in J \cap D(J)$, then the return domain $L$ to $J$ containing $x$ is well inside the return domain $L^{\prime}$ to $I^{\prime}$ containing $x$ by Proposition 15 , Since $\partial J \cap D\left(I^{\prime}\right)=\emptyset$, we obtain $L^{\prime} \subset J$. Then $L$ is well inside $J$ and $J$ is uniformly nice.

Claim 2. Let $c_{1}, c_{2}, \ldots, c_{k} \in$ Crit and $V_{i} \ni c_{i}$ be nice intervals such that $V=\bigcup_{i=1}^{k} V_{i}$ is a $\xi$-nice set and $\partial V \subset Z$. Then there is $\xi^{\prime}=\xi^{\prime}(\xi)>0$ such that the following hold.

(1) For each $1 \leq i \leq k$, there exist nice intervals $W_{i} \supset \tilde{V}_{i} \ni c_{i}$ such that

- $\tilde{V}_{i}$ is $\xi^{\prime}$-well inside $W_{i}$ and $W_{i}$ is $\xi^{\prime}$-well inside $V_{i}$;

- $\partial \tilde{V}_{i} \cap D(V)=\emptyset$ and $\partial W_{i} \cap D(V)=\emptyset$. In particular, $\bigcup_{i=1}^{k} \tilde{V}_{i}$ is a nice set;

(2) For each $x \in V_{i} \backslash \mathrm{Cl} \tilde{V}_{i}$, there is a nice interval $J_{x} \ni x$ such that $J_{x}$ is $\xi^{\prime}$-well inside $V_{i}$ and $J_{x} \cap \tilde{V}_{i}=\emptyset, \partial J_{x} \cap D(V)=\emptyset$.

(3) The endpoints of all intervals above belong to $Z$.

Claim 2 is exactly [CL, Lemma 3], except that we additionally request $\partial V \subset Z$ and get the extra property (3) in return. The proof requires no changes: only, instead of defining the auxiliary interval $\left(p^{\prime}, q^{\prime}\right)=\left(a-\xi^{\prime}(b-a) / 4, b+\xi^{\prime}(b-a) / 4\right)$ for $\tilde{V}_{i}=(a, b)$, we choose $p^{\prime}, q^{\prime} \in Z$ with $p^{\prime} \in\left(a-\xi^{\prime}(b-a) / 3, a-\xi^{\prime}(b-a) / 4\right)$ and $q^{\prime} \in\left(b+\xi^{\prime}(b-a) / 4, b+\xi^{\prime}(b-a) / 3\right)$.

We are now in position to prove Proposition 45. This is done inductively. Let Crit $\backslash$ Feig $=$ $\left\{c_{1}, \ldots, c_{m}\right\}$. If $m=1$, then this is just Claim 1. Assume that we have constructed intervals $V_{i} \ni c_{i}$ with $\left|V_{i}\right|<\varepsilon$ and $\partial V_{i} \subset Z, 1 \leq i \leq k$, such that $\bigcup_{i=1}^{k} V_{i}$ is $\xi_{k}$-nice for some constant $\xi_{k}>0$. We will show that there are smaller intervals $\tilde{V}_{i} \ni c_{i}$ with $\partial \tilde{V}_{i} \subset Z$, and a constant $\xi_{k+1}$ depending only on $\xi_{k}$, such that $\bigcup_{i=1}^{k+1} \tilde{V}_{i}$ is $\xi_{k+1}$-nice.

The intervals $\tilde{V}_{i}, 1 \leq i \leq k$, are those from Claim 2. To define $\tilde{V}_{k+1}$ and conclude the proof, two cases must be considered. If $c_{k+1} \in D\left(\bigcup_{i=1}^{k} V_{i}\right)$, then Cai and Li's proof works without any changes (it uses Claim 2 in its full extension).

If $c_{k+1} \notin D\left(\bigcup_{i=1}^{k} V_{i}\right)$, then we need to find intervals $c_{k+1} \subset \tilde{V}_{k+1} \subset V_{k+1}$ with $\left|V_{k+1}\right|<\varepsilon$, $\tilde{V}_{k+1}$ well inside $V_{k+1}$ and $\partial \tilde{V}_{k+1} \subset Z$, such that $\bigcup_{i=1}^{k+1} V_{i}$ is nice and $\partial \tilde{V}_{i} \notin D\left(\bigcup_{i=1}^{k+1} V_{i}\right)$ for every $1 \leq i \leq k+1$.

We define $V_{k+1}$ and $\tilde{V}_{k+1}$. Since $\tilde{V}_{k+1}$ is well inside $V_{k+1}$ and $\partial \tilde{V}_{k+1} \subset Z$, we only need to show that $\partial V_{k+1}, \partial \tilde{V}_{k+1} \notin D\left(\bigcup_{i=1}^{k+1} V_{i}\right)$. (Recall that the endpoints of the intervals $V_{i}, \tilde{V}_{i}$, $i \leq k$, belong to $Z$, hence their orbits cannot visit $V_{k+1}$ if it is sufficiently small.)

As in the proof of Claim 1, three possibilities arise for $c_{k+1}$. The simplest case is when $c_{k+1}$ is solenoidal. Then $V_{k+1}$ is defined as in Claim 1 and $\tilde{V}_{k+1}$ is the return domain to $V_{k+1}$ containing $c_{k+1}$; everything works because $c_{k+1} \notin D\left(\bigcup_{i=1}^{k} V_{i}\right)$.

Now assume that $c_{k+1}$ is not solenoidal. Starting from a small interval $\left(a^{\prime}, b^{\prime}\right) \ni c_{k+1}$ with $a^{\prime}, b^{\prime} \in Z$ and repeating the reasoning in Case 2 of Cai and Li's proof, we find an interval $c_{k+1} \in(a, b) \subset\left(a^{\prime}, b^{\prime}\right)$ such that $a, b \in Z$ and $(a, b) \cup \bigcup_{i=1}^{k} V_{i}$ is nice. If $c_{k+1}$ is recurrent, we take $I_{0}=(a, b)$ in Proposition [16, and define accordingly the intervals $I_{m}$. Then $I_{m} \cup \bigcup_{i=1}^{k} V_{i}$ is nice for every $m$ because $c_{k+1} \notin D\left(\bigcup_{i=1}^{k} V_{i}\right)$. Now it suffices to fix $m^{\prime}$ such that $I_{m^{\prime}}$ is uniformly nice and define $V_{k+1}=I_{m^{\prime}}$ and $\tilde{V}_{k+1}=I_{m^{\prime}+1}$. If $c_{k+1}$ is 
non-recurrent, then we find an interval $J \ni c_{k+1}$, similarly as we did in Claim 1, such that $\partial J \subset Z, \partial J \cap D\left((a, b) \cup \bigcup_{i=1}^{k} V_{i}\right)=\emptyset$, and $J$ is $1 / 2$-well inside $(a, b)$. Then $V_{k+1}=(a, b)$ and $\tilde{V}_{k+1}=J$ are adequate to our purposes.

Let us finally show that with this choice, $\tilde{V}=\bigcup_{i=1}^{k+1} \tilde{V}_{i}$ is uniformly nice. Let $x \in \tilde{V}_{n} \cap D(\tilde{V})$, say $\phi_{\tilde{V}}(x) \in \tilde{V}_{l}$. Let $0 \leq s \leq t$ be the entry time of $x$ to $\tilde{V}_{l}$ and the return time of $x$ to $\tilde{V}$, respectively, and denote the return domain to $\tilde{V}$ and the entry domain to $V_{l}$ containing $x$ by $J$ and $K$ respectively, so $x \in J \subset K$. By Proposition 15, the pullback $H$ of $\tilde{V}_{l}$ along $f^{s}(x), \ldots, f^{t}(x)$ is well inside $V_{l}$. Note that $J$ is the pullback of $H$ along $x, \ldots, f^{s}(x)$ and $K$ is the pullback of $V_{l}$ along $x, \ldots, f^{s}(x)$. Hence $J$ is well inside $K$ by Corollary 14, Since $\partial \tilde{V}_{n} \notin D\left(\bigcup_{i=1}^{k+1} V_{i}\right), K$ is contained in $\tilde{V}_{n}$, so $J$ is well inside $\tilde{V}_{n}$. This proves that $\tilde{V}$ is uniformly nice.

Proof of Theorem 20. Fix $\varepsilon>0$. We define the sets $c \in U_{c} \subset V_{c}$ and $W_{c}$ as follows. If $c \in$ Crit $\backslash$ Feig, then $V_{c}$ is the component of the set $V$ from Proposition 45 containing $c$. Write $V_{c}=(a, b)$ and say, for instance, that $(c, b)$ is the smallest component of $V_{c} \backslash\{c\}$. Define the convex combinations

$$
v_{0}:=\frac{c+\xi_{0}^{\prime} b}{1+\xi_{0}^{\prime}} \quad \text { and } \quad v_{i}:=\frac{v_{i-1}+\xi_{0}^{\prime} b}{1+\xi_{0}^{\prime}}, \quad i=1,2,3
$$

with $\xi_{0}^{\prime}=\xi_{0}(f) / 2$ and $\xi_{0}(f)$ the constant from Proposition 45. Take $v^{\prime} \in\left(v_{0}, v_{1}\right) \cap Z$ close to $v_{0}$. If $v^{\prime} \notin D(V)$, then set $v=v^{\prime}$; otherwise let $v$ be the right endpoint of the return domain to $V$ containing $v^{\prime}$, and by having chosen $v^{\prime}$ close to $v_{0}$, we obtain $v_{0}<v^{\prime} \leq v<v_{1}$. This gives $v \in\left(\left(v_{0}, v_{1}\right) \cap Z\right) \backslash D(V)$, and, similarly, there is $w \in\left(\left(v_{2}, v_{3}\right) \cap Z\right) \backslash D(V)$. If $c \in D(V)$, let $u$ denote the left endpoint of the return domain to $V$ containing $c$; if $c \notin D(V)$, take $u_{0}=c-\left(v_{0}-c\right)=2 c-v_{0}$ and find as before $u \in\left(u_{0}, c\right)$ belonging to $Z \backslash D(V)$. Finally we define $U_{c}=(u, w)$ and $W_{c}=(v, w)$.

For critical points of Feigenbaum type we rely on Proposition 43, More precisely, for every Feigenbaum solenoidal set $S$, we find a minimal solenoidal cycle $T=\operatorname{cyc}(\hat{J})=\bigcup_{i=0}^{s-1} f^{i}(\hat{J})$ containing $S$ whose constituting intervals are small enough to ensure that $\left|V_{c}\right|<\varepsilon$ for the components of the corresponding set $V$. Then the intervals $U_{c}$ are those from the shell $M=\bigcup_{i=0}^{s-1} M_{i}$ of $T$ containing points from Crit (that is, from Feig). To define the sets $V_{c}$ assume, after reordering, that $M_{0}=M_{s}$ is the smallest of all intervals $M_{i}$. Since the intervals $f^{i}(\hat{J})$ are well centered in the intervals $M_{i}$ by Proposition 43, there is an interval $G_{s}$ such that $M_{s}$ is well inside $G_{s}$ and $G_{s}$ intersects no other interval from the solenoidal cycle $T$ than $\hat{J}=f^{s}(\hat{J})$. Pulling back $G_{s}$ along $f(\hat{J}), \ldots, f^{s}(\hat{J})$, we construct similarly intervals $G_{i}, i=1, \ldots, s$, such that $M_{i}$ is well inside $G_{i}$ and $G_{i} \cap T=f^{i}(\hat{J})$. The intervals $V_{c}$ are those intervals $G_{i}$ containing points from Crit.

To define the sets $W_{c}$ we take a boundary point $q$ of $M_{0}$ with $f^{s}(q)=q$ (in fact the proof of Proposition 43 shows that it is possible that $f^{s / 2}(q)=q$ ). For every $0 \leq i<s$, let $u_{i}$ denote the middle point between $f^{i}(q)$ and the endpoint of $f^{i}(\hat{J})$ closest to $f^{i}(q)$. If $M_{i}$ contains a critical point, let $L_{i}$ be the interval with endpoints $f^{i}(q)$ and $u_{i}$; if not, let $L_{i}=M_{i}$. Finally, let $L_{i}^{\prime} \subset L_{i}$ be the largest interval with endpoint $f^{i}(q)$ such that $f^{j}\left(L_{i}^{\prime}\right) \subset L_{i+j}$ for all $0 \leq j<s$ with indices taken $\bmod s$, and hence $\left.f^{s}\right|_{L_{i}^{\prime}}$ is a diffeomorphism. Now the intervals $W_{c}$ are those intervals $L_{i}^{\prime}$ contained in intervals $U_{c}=M_{i}$ intersecting Crit. 
We show that (i)-(iii) in Theorem 20 hold. Clearly, the construction implies the existence of a number $\xi=\xi(f)>0$, thus not depending on $\varepsilon$, such that $U_{c}$ is $\xi$-well inside $V_{c}$ for every $c \in$ Crit. Moreover, since the endpoints of all sets $W_{c^{\prime}}, U_{c^{\prime}}, V_{c^{\prime}}, c^{\prime} \in$ Crit $\backslash$ Feig, belong to $Z \backslash D(V)$, we can assume that they do not belong to $D\left(V_{c}\right), c \in$ Feig, either. If $c^{\prime} \in$ Feig, then the niceness and invariance of shells still guarantees $\partial U_{c^{\prime}} \cap D\left(U_{c}\right)=\emptyset$ for every $c \in$ Crit and $\partial U_{c^{\prime}} \cap D\left(V_{c}\right)=\emptyset$ for every $c \in$ Crit not belonging to the same solenoidal set as $c^{\prime}$. In particular, $U=\bigcup_{c \in \text { Crit }} U_{c}$ is nice. This proves Theorem 20(i).

Now let $J$ be an entry domain to $U$, say $\left.\phi\right|_{J}=\left.f^{j}\right|_{J}$ and $\phi(J)=U_{c}$. Then $\left.f^{j}\right|_{J}$ is a diffeomorphism. We want to show that $\left.f^{j}\right|_{J}$ extends to a diffeomorphism $\left.f^{j}\right|_{K}: K \rightarrow V_{c}$. Assume by contradiction that this is not the case. Then there is $K^{\prime} \supset J$ such that $\left.f^{j}\right|_{K^{\prime}}$ is a diffeomorphism, one of the endpoints $a$ of $K^{\prime}$ satisfies $c^{\prime}=f^{n}(a) \in$ Crit for some $1 \leq n<j$, and $f^{j}\left(\{a\} \cup K^{\prime}\right) \subset V_{c}$.

Let $b \in K^{\prime}$ such that $f^{n}(b) \in \partial U_{c^{\prime}}$ (here we use that $f^{n}(J)$ does not intersect $U$ ). We can assume that both $c$ and $c^{\prime}$ belong to the same Feigenbaum solenoidal set $S$, because otherwise $\partial U_{c^{\prime}} \cap D\left(V_{c}\right)=\emptyset$, which contradicts $f^{j}(b) \in V_{c}$. Let $T=\operatorname{cyc}(\hat{J})$ be the minimal solenoidal cycle for $S$ we used earlier to construct the sets $U_{c}, V_{c}$. Then there is $i \in \mathbb{N}$ such that $f^{j}(J)=U_{c} \supset f^{i}(\hat{J})$. Since $f^{j}(a)=f^{j-n}\left(c^{\prime}\right) \in S$, there is $i^{\prime} \in \mathbb{N}$ such that $f^{j}(a) \in f^{i^{\prime}}(\hat{J})$. But $\left.f^{j}\right|_{\{a\} \cup K^{\prime}}$ is a homeomorphism, so $f^{i}(\hat{J})$ and $f^{i^{\prime}}(\hat{J})$ are different. This is impossible, because by its definition $V_{c}$ intersects exactly one interval from $T$.

We have shown that if $J$ is an entry domain to $U$, then $\left.f^{j}\right|_{J}: J \rightarrow U_{c}$ extends to a diffeomorphism $\left.f^{j}\right|_{K}: K \rightarrow V_{c}$. Since $U_{c}$ is well inside $V_{c}$, there is $\kappa=\kappa(f)$ such that $\left.f^{j}\right|_{J}$ has distortion bounded by $\kappa$ by the $C^{2}$ Koebe Principle (Proposition 15). This finishes the proof of Theorem 20)(ii).

It remains to prove Theorem 20(iii). If $c \in$ Crit $\backslash$ Feig, then the definition of $W_{c}$ easily implies that it is not too small compared to $U_{c}$. On the other hand, it is not too large compared to the subinterval of $U_{c}$ between $c$ and $W_{c}$, so $\left.f\right|_{W_{c}}$ has bounded distortion because $c$ is non-flat. Since $\partial W_{c} \cap D(U)=\emptyset$, Theorem 20(iii) holds in this case.

Assume now that $c \in$ Feig and $U_{c}$ is one of the above intervals $M_{i_{1}}$, with $W_{c}=L_{i_{1}}^{\prime}$. Put $k_{c}=s$ and let $i_{1}<i_{2}<\cdots<i_{t}<i_{1}+s$ be the indices $i$ such that $M_{i}$ contains some critical point. As shown in the proof of Proposition 43, each map $\left.f^{i_{r+1}-i_{r}-1}\right|_{M_{i_{r}+1}}$ has bounded distortion. Also, each map $\left.f\right|_{L_{i_{r}}}$ has bounded distortion because $\left|L_{i_{r}}\right|$ is less than half the distance from $f^{i_{r}}(q)$ to the critical point in $M_{i_{r}}$ and non-flatness applies. Hence $\left.f^{k_{c}}\right|_{W_{c}}=\left.f^{s}\right|_{L_{i_{1}}^{\prime}}$ has bounded distortion. Recall that all periodic orbits in the intervals $M_{i}$ are repelling, and the same is true for $\operatorname{orb}(q)$. Then $f^{k_{c}}\left(W_{c}\right) \supset W_{c}$.

Finally, let us show that $W_{c}$ is not too small compared to $U_{c}$. From its definition, $L_{i_{1}}$ is not too small compared to $U_{c}$. Let $A_{j}$ denote the largest interval with endpoint $f^{j}(q)$ such that $f^{k}\left(A_{j}\right) \subset L_{j+k}$ for all $0 \leq k \leq i_{j}-i_{j-1}$; hence $A_{1}=L_{i_{1}}$ and $A_{t}=W_{c}$. We claim that $A_{j+1}$ is not too small compared with $A_{j}$. Indeed, $L_{i_{j+1}}$ is not too small compared to $M_{i_{j+1}}$, so a fortiori is not too small compared to $f^{i_{j+1}-i_{1}}\left(A_{j}\right)$. Since $\left.f^{i_{j+1}-i_{1}}\right|_{A_{j}}$ has bounded distortion and $A_{j+1}=A_{j} \cap f^{-\left(i_{j+1}-i_{1}\right)}\left(L_{i_{j+1}}\right), A_{j+1}$ cannot be too small compared to $A_{j}$ either.

This concludes the proof of Theorem 20 . 


\subsection{A $C^{3}$ Koebe Distortion Lemma.}

Lemma 46. Let $f \in C_{\mathrm{nf}}^{3}(I)$. Then for any $\xi>0$ and $k \geq 0$, there is $\xi^{\prime}=\xi^{\prime}(\xi, k, f)>0$ such that the following statement holds: Let $\left(H_{i}\right)_{i=0}^{l} \subset\left(G_{i}\right)_{i=0}^{l}$ be chains such that $\left(G_{i}\right)_{i=0}^{l}$ has order at most $k$ and $G_{l}$ is a small nice interval close enough to Crit. If $H_{l}$ is $\xi$-well inside $G_{l}$, then $H_{0}$ is $\xi^{\prime}$-well inside $G_{0}$. Moreover, if $k=0$, then there is $\kappa=\kappa(\xi, f)>0$ such that $\left.f^{l}\right|_{H_{0}}$ has distortion bounded by $\kappa$.

A slightly weaker version of this lemma (requiring that the intervals $G_{i}$ are not too close to parabolic periodic points) appears in [LS], who in turns refer to [SV, Theorem $\mathrm{C}(2)]$. Our proof is based on [SV, Proposition 3] and Theorem 20.

Proof of Lemma 46. Assuming that the components of $U$ in Theorem 20] are sufficiently small, we can conclude that iterates of $f$ one beyond those mapping into $U$ have negative Schwarzian derivative. The idea behind obtaining negative Schwarzian derivative goes back to Kozlovski [Ko, see also [GSS], and the precise statement is as follows.

Let $f \in C_{\mathrm{nf}}^{3}(I)$ and $U$ be as in Theorem 20. If the components $U_{c}$ of $U$ are sufficiently small, and $x$ is such that $f^{n}(x) \in U$ and $f^{i}(x) \notin$ Crit for every $0 \leq i \leq n$, then the Schwarzian derivative of $f^{n+1}$ at $x$ is negative.

"Sufficiently small" in this statement should be interpreted as that for each component $U_{c}$ of $U$ and each $i \geq 0$, each component of $f^{-i}\left(U_{c}\right)$ has length $\leq \tau$, where $\tau=\tau(f)$ is taken from [SV, Proposition 3] (choosing, with the notation in [SV, Proposition 3], $S=1, N=0$ and $\delta$ the number $\xi_{0}=\xi(f)$ from Theorem 20). If $U_{c}$ is sufficiently small, then this holds by Proposition [7. To prove the statement, let $\left(J_{i}\right)_{i=0}^{n}$ be the pullback chain of $U_{c}$ along $x, f(x), \ldots, f^{n}(x) \in U_{c}$. Let $t_{1}<t_{2}<\cdots<t_{m}$ be the iterates such that $f^{t_{j}}(x) \in U$, and hence the intervals $J_{i}, t_{j}+1 \leq i \leq t_{j+1}$ are pairwise disjoint and $\sum_{i=t_{j}+1}^{t_{j+1}}\left|J_{i}\right| \leq 1$. Fix $j>1$ for the moment. Then $J_{t_{j}+1}$ is contained in an entry domain $J$ to $U$, say $f^{t_{j+1}-t_{j}-1}(J)=U_{c^{\prime}}$. By Theorem 20(ii), there is $K \supset J$ such that $f^{t_{j+1}-t_{j}-1}$ maps diffeomorphically $K$ onto $V_{c^{\prime}}$, hence the pullback chain of $V_{c^{\prime}}$ along $J_{t_{j}+1}, \ldots, J_{t_{j+1}}$ has order 0 . Moreover, $J_{t_{j+1}}$ is contained in $U_{c^{\prime}}$, so it is $\xi_{0}$-well inside $V_{c^{\prime}}$. Therefore [SV, Proposition 3] implies that $f^{t_{j+1}-t_{j}}$ has negative Schwarzian derivative at $f^{t_{j}+1}(x)$. For $j=1$ and $t_{1}>0$ (so $\left.x \notin U\right), f^{t_{1}+1}$ has negative Schwarzian derivative at $x$ by the same reasoning. If $t_{1}=0$ (so $x \in U$ ), then $f$ has negative Schwarzian derivative at $x$ by non-flatness. Since compositions of maps with negative Schwarzian derivatives have negative Schwarzian derivative, the statement follows.

Now we continue with the proof of the lemma. Assume first $k=0$. Fix $\xi>0$ and let $\left(H_{i}\right)_{i=0}^{l} \subset\left(G_{i}\right)_{i=0}^{l}$ be chains such that $\left(G_{i}\right)_{i=0}^{l}$ has order 0 and $G_{l}$ is contained in a component $U_{c}$ of the set $U$ above. If $G_{0}$ is an entry domain to $G_{l}$, then the statement is just Proposition 13 because the intervals $G_{i}$ are pairwise disjoint ( $G_{l}$ is nice). If not, again due to the niceness of $G_{l}$, there is an interval $G_{t} \subset G_{l}$ such that the intervals $G_{t+1}, \ldots, G_{l}$ are pairwise disjoint, so we can apply Proposition 13 to the subchains $\left(H_{i}\right)_{i=t+1}^{l} \subset\left(G_{i}\right)_{i=t+1}^{l}$. Also, $\left.f^{t+1}\right|_{G_{0}}$ has negative Schwarzian derivative by the above statement, so we can apply Proposition 12 to the subchains $\left(H_{i}\right)_{i=0}^{t+1} \subset\left(G_{i}\right)_{i=0}^{t+1}$. 
The general case $k>0$ follows easily from this one (take also Lemma 11 into account). Now we need $G_{l}$ to be small enough so that every component of every preimage of $G_{l}$ is contained in $U$ whenever it intersects Crit (again by Proposition 7 ).

7.4. Proof of Theorem 21. Let $x \in I$ be such that $\operatorname{orb}(x)$ is disjoint from $\partial I$. For every $n \in \mathbb{N}$ and every $0<\varepsilon<d\left(f^{n}, \partial I\right)$ we construct the pullback chain of $\left(f^{n}(x)-\varepsilon, f^{n}(x)+\varepsilon\right)$ along $x, f(x), \ldots, f^{n}(x)$. We define

$$
r_{n}^{k}(x)=\sup \left\{\varepsilon>0: \text { order of the pullback chain of }\left(f^{n}(x)-\varepsilon, f^{n}(x)+\varepsilon\right) \leq k\right\} .
$$

If for every $k$ we have $r_{n}^{k}(x) \rightarrow 0$ as $n \rightarrow \infty$, then we call $x$ a super-persistent point. In [BM1, Theorem 2.7] (see also [BM2]) it is shown that for $f \in C_{\mathrm{nf}}^{2}(I)$, the $\omega$-limit set of any super-persistent recurrent point of $f$ is minimal.

Proof of Theorem [21. By definition of $E$ and type (2) attractors, $\lambda$-a.e. $x \in E$ has a dense orbit in some cycle $\operatorname{cyc}(K)$ and therefore cannot be super-persistently recurrent, nor map into $\partial I$. We can then find a sequence $n_{j} \rightarrow \infty$ and $N_{x} \in \mathbb{N}$ and $\delta_{x}>0$ such that the pullback chain of $\left(f^{n_{j}}(x)-\delta_{x}, f^{n_{j}}(x)+\delta_{x}\right)$ along $x, \ldots, f^{n_{j}}(x)$ has order at most $N_{x}$. Clearly we can take $\delta_{f(x)} \geq \delta_{x}$ and $N_{f(x)} \leq N_{x}$ (in fact, we have equality for large $j$ whenever $x$ is not a critical point). Since Lebesgue measure has only finitely many ergodic components [Ly1, Theorem 2], it follows that there are a single $\delta>0$ and $N \in \mathbb{N}$ such that $\delta_{x} \geq \delta$ and $N_{x} \leq N$ for $\lambda$-a.e. $x \in E$.

Next choose $\varepsilon>0$ in Theorem 20 so small that the intervals $U_{c} \subset V_{c}, c \in \mathrm{Crit}^{\prime}$, satisfy:

- if $c$ is in the interior of the metric attractor $\operatorname{cyc}(K) \mathrm{r}$, then $\operatorname{dist}\left(V_{c}, \partial \operatorname{cyc}(K)\right)>2 \delta$; moreover, if $d \in$ Crit $\backslash$ Crit' $^{\prime}$, then either $d \in \partial \operatorname{cyc}(K)$ or $\operatorname{dist}(d$, $\operatorname{cyc}(K))>\delta$;

- every component of every preimage of $V_{c}$, and every image $f^{n}\left(V_{c}\right), n \leq 2 r$ (with $r$ the period of $\operatorname{cyc}(K))$, has length less than $\delta$.

Recall that $U^{\prime}=\bigcup_{c \in \mathrm{Crit}^{\prime}} U_{c}, V^{\prime}=\bigcup_{c \in \mathrm{Crit}^{\prime}} V_{c}$. From their definition, both sets are nice. Moreover, $\partial U^{\prime} \cap D\left(V^{\prime}\right)=\emptyset$ where as before $D\left(V^{\prime}\right)=\cup_{n>0} f^{-n}\left(V^{\prime}\right)$.

Since $\operatorname{orb}(x)$ is dense in $\operatorname{cyc}(K)$, we can assume that the numbers $n_{j}$ are large enough so that $f^{n_{j}}(x) \in \operatorname{cyc}(K)$ for every $j$. Let $J$ be the entry domain to $U$ (or the component of $U$ ) containing $f^{n_{j}}(x)$, say $f^{m_{j}}(J)=U_{c}$. Two possibilities arise. If $J \subset \operatorname{cyc}(K)$, then

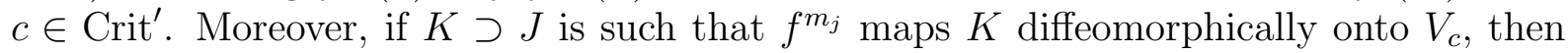
$K \subset\left(f^{n_{j}}(x)-\delta, f^{n_{j}}(x)+\delta\right)$, so the pullback chain of $V_{c}$ along $x, \ldots, f^{n_{j}+m_{j}}(x)$ has order at most $N$. The second possibility is that $J$ contains some point from $\partial \operatorname{cyc}(K)$, call it $a$. It is not possible that $a$ belongs to a periodic orbit contained in $\partial \operatorname{cyc}(K)$, because this would imply that one of the points of this periodic orbit belongs to Crit $\backslash$ Crit $^{\prime}$, which contradicts that $\operatorname{cyc}(K)$ contains dense orbits. Hence there is $r_{j} \leq 2 r$ (with $r$ the period of

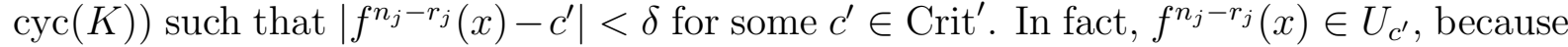
otherwise $f^{n_{j}-r_{j}}(x)$ would belong to an entry domain $J^{\prime}$ to $U$ contained in $\operatorname{cyc}(K)$, which leads to the contradiction $f^{r_{j}}\left(J^{\prime}\right)=J \subset \operatorname{cyc}(K)$. Since $f^{r_{j}}\left(V_{c^{\prime}}\right) \subset\left(f^{n_{j}}(x)-\delta, f^{n_{j}}(x)+\delta\right)$, the pullback chain of $V_{c^{\prime}}$ along $x, \ldots, f^{n_{j}-r_{j}}(x)$ has order at most $N$.

We have proved that there is a number $N=N(f)$ such that, for a.e $x \in D$, there are $c \in$ Crit $^{\prime}$ and a sequence $s_{j} \rightarrow \infty$ such that $f^{s_{j}}(x) \in U_{c}$ and the pullback of $V_{c}$ along 
$x, \ldots, f^{s_{j}}(x)$ has order at most $N$. However, we need critical order 0 , not $N$, for this theorem, so a further argument is required.

Let $k_{x} \geq 1$ be the smallest integer for which there are $G_{x} \supset H_{x} \ni x$ and $c \in$ Crit' $^{\prime}$ such that $f^{k_{x}}$ maps $G_{x}$ diffeomorphically onto $V_{c}$ and $f^{k_{x}}\left(H_{x}\right)=U_{c}$. If $x \notin U^{\prime}, \omega(x)=\operatorname{cyc}(K)$ and the entry domain $H_{x} \ni x$ to $U^{\prime}$ is contained in cyc $(K)$, then $k_{x}$ exists; it is the first entry time $k_{x}=r_{U^{\prime}}(x)$. But if $x \in U^{\prime}$, then $k_{x}$ is more difficult to find.

Claim 1. The set $B:=\left\{x \in E: k_{x}\right.$ exists $\}$ has full Lebesgue measure in $E$.

Assume by contradiction that this claim fails, and that $x$ is a density point of $E \backslash B$. We can find the sequence $\left(s_{j}\right)_{j \in \mathbb{N}}$ and $c \in$ Crit $^{\prime}$ as above, and let $\left(G_{i}^{j}\right)_{i=0}^{s_{j}}$ and $\left(H_{i}^{j}\right)_{i=0}^{s_{j}}$ be the pullback chains of $G_{s_{j}}^{j}=V_{c}$ and $H_{s_{j}}^{j}=U_{c}$ along $x, \ldots, f^{s_{j}}(x)$, respectively. The chains $\left(G_{i}^{j}\right)_{i=0}^{s_{j}}$ have order $\leq N$.

Let $\left(W_{t}\right)_{t \in \mathbb{N}}$ be a denumeration of the return domains to $V^{\prime}$ within $V_{c} \backslash U_{c}$ and also take $W_{0}:=U_{c}$. Recall that $\partial U_{c} \cap D\left(V^{\prime}\right)=\emptyset$, so $\left(W_{t}\right)_{t \geq 0}$ is a family of pairwise disjoint intervals whose union has full measure in $V^{\prime}$. Since $\left.f^{s_{j}}\right|_{G_{0}^{j}}$ has at most $N$ critical values, we can arrange the enumeration of the $W_{t}$ s such that no critical value of $\left.f^{s_{j}}\right|_{G_{0}^{j}}$ is contained in $W_{t}$ if $t>N$. By Proposition 45 there is $\xi=\xi(f)$ such that each $W_{t}$ is $\xi$-well inside $V_{c}$. By Lemma 46, there is $\zeta=\zeta(\xi, N, f)$ such that each component of $G_{0}^{j} \cap f^{-s_{j}}\left(\bigcup_{t=0}^{N} W_{t}\right)$ is $\zeta$-well inside $G_{0}^{j}$. This holds in particular for the at most $M \leq 2^{N+1}-1$ components of $f^{-s_{j}}\left(\bigcup_{t=0}^{N} W_{t}\right)$ in $G_{0}^{j}$. Let us denote these components as $Y_{i}$, numbered in order of decreasing size.

Claim 2. There are $b=b(\zeta, N)>0$ and $B^{j} \subset G_{0}^{j}$ such that $\lambda\left(B^{j}\right) \geq b \lambda\left(G_{0}^{j}\right)$ and for every $y \in B^{j}$, there are $k_{y} \geq s_{j}, G_{y} \supset H_{y} \ni y$ and $c(y) \in$ Crit $^{\prime}$ such that $f^{k_{y}}$ maps $G_{y}$ diffeomorphically onto $V_{c(y)}$ and $f^{k_{y}}\left(H_{y}\right)=U_{c(y)}$.

Take $b=\zeta^{M} /(M+1)$ !. The components $Y_{i}$ are pairwise disjoint, and have $\zeta$-collars around them in $G_{0}^{j}$, which may intersect other components $Y_{i^{\prime}}$ or their collars. We will show that $\lambda\left(\bigcup_{i} Y_{i}\right) \leq(1-b) \lambda\left(G_{0}^{j}\right)$. If $\lambda\left(Y_{1}\right)<\lambda\left(G_{0}^{j}\right) /(M+1)$, then we get directly

$$
\lambda\left(\bigcup_{i} Y_{i}\right)<\lambda\left(G_{0}^{j}\right) M /(M+1)=(1-1 /(M+1)) \lambda\left(G_{0}^{j}\right)<(1-b) \lambda\left(G_{0}^{j}\right) .
$$

Thus we can assume $\lambda\left(Y_{1}\right) \geq \lambda\left(G_{0}^{j}\right) /(M+1)$ and the $\zeta$-collar of $Y_{1}$ has two components of length $\geq \zeta \lambda\left(G_{0}^{j}\right) /(M+1)$. If the second largest $Y_{2}$ satisfies $Y_{2}<\zeta \lambda\left(G_{0}^{j}\right) /((M+1) M)$, then there is a set in the $\zeta$-collar of $Y_{1}$ of measure $>\zeta \lambda\left(G_{0}^{j}\right) /((M+1) M)$ which do not intersect $\bigcup_{i} Y_{i}$. Hence

$$
\lambda\left(\bigcup_{i} Y_{i}\right)<(1-\zeta /((M+1) M)) \lambda\left(G_{0}^{j}\right)<(1-b) \lambda\left(G_{0}^{j}\right)
$$

again. Continuing this way, we find that at least one component $Y_{k}$ has a $\zeta$-collar disjoint from $\bigcup_{i} Y_{i}$ and its size is $\geq \zeta^{M} \lambda\left(G_{0}^{j}\right) /(M+1) !=b \lambda\left(G_{0}^{j}\right)$.

Now take $B^{j}:=E \cap\left(G_{0}^{j} \backslash \bigcup_{i} Y_{i}\right)$. Then clearly $\lambda\left(B^{j}\right) \geq b \lambda\left(G_{0}^{j}\right)$, and if $y \in B^{j}$, we find $k_{y}$ as follows. We have $z:=f^{s_{j}}(y) \in W_{t}$ for some $t>N$. There is $r_{t}$ such that $f^{r_{t}}$ maps $W_{t}$ diffeomorphically onto a component of $V^{\prime}$. If $f^{r_{t}}(z) \in U^{\prime}$, then $k_{y}=s_{j}+r_{t}$. Otherwise $f^{r_{t}}(z) \in V^{\prime} \backslash U^{\prime}$ and in fact $f^{r_{t}}(z)$ belong to another return domain to $V^{\prime}$. We continue 
iterating diffeomorphically until finally $z$ falls into $U^{\prime}$, and we choose $k_{y}$ accordingly. This proves the Claim 2. Obviously $B^{j} \subset B$, so $\lambda\left(B \cap G_{0}^{j}\right) \geq b \lambda\left(G_{0}^{j}\right)$ independently of $j$. Since $\lambda\left(G_{0}^{j}\right) \rightarrow 0$ as $j \rightarrow \infty$, this contradicts that $x$ is a density point of $I \backslash B$, proving Claim 1 .

From Claim 1 the first part of Theorem 21 easily follows: if $x, y \in B$ and $k_{x} \leq k_{y}$, then either $H_{x} \cap H_{y}=\emptyset$ or $H_{y} \subset H_{x}$. Now an easy maximality argument allows to redefine these sets if necessary so that either $H_{x} \cap H_{y}=\emptyset$ or $H_{y}=H_{x}$.

It remains to prove the second part of Theorem 21. Since a.e. point from $U^{\prime}$ belongs to $B, F: U^{\prime} \rightarrow U^{\prime}$ is well defined a.e. It is important to note that if $F$ is defined on $x$, then $H_{x} \subset G_{x} \subset U^{\prime}$ because $\partial\left(U^{\prime}\right) \cap D\left(V^{\prime}\right) \neq \emptyset$. Now it is immediate so show by induction on $n$ that every branch $\left.F^{n}\right|_{H}=\left.f^{j}\right|_{H}: H \subset U \rightarrow U_{c}$ of $F^{n}$ admits a diffeomorphic extension to an interval $H \subset G \subset U$ with $f^{j}(G)=V_{c}$. Then $\left.F^{n}\right|_{H}$ has distortion bounded by a constant $\kappa$ depending neither on $n$ nor on $H$ by Lemma 46 ,

\section{REFERENCES}

[AJS] L. Alsedà, V. Jiménez López, L. Snoha, All solenoids of piecewise smooth maps are period doubling, Fund. Math. 157 (1998) 121-138.

[BJ1] F. Balibrea, V. Jiménez López, A structure theorem for $C^{2}$ functions verifying the Misiurewicz condition, in: Proceedings of the European Conference on Iteration Theory (Lisbon, 1991), 12-21, World Sci. Publishing, Singapur, 1992.

[BJ2] F. Balibrea, V. Jiménez López, The measure of scrambled sets: a survey, Acta Univ. M. Belii Ser. Math. No. 7 (1999) 3-11.

[BD] M. Barge, B. Diamond, Proximality in Pisot tiling spaces, Fund. Math. 194 (2007) 191-238.

[Ba] J. A. Barnes, Conservative exact rational maps of the sphere, J. Math. Anal. Appl. 230 (1999) 350-374.

[BrJ] A. Barrio Blaya, V. Jiménez López, An almost everywhere version of Smítal's order-chaos dichotomy for interval maps, J. Austral. Math. Soc. 85 (2008) 29-50.

[BHS] F. Blanchard, W. Huang, L. Snoha, Topological size of scrambled sets, Colloq. Math. 110 (2008) 293-361.

[Bl] L. S. Block, Stability of periodic orbits in the theorem of Šarkovskii, Proc. Amer. Math. Soc. 81 (1981) 333-336.

[BC] L. S. Block, W. A. Coppel, Dynamics in one dimension, Lecture Notes in Mathematics, 1513, Springer-Verlag, Berlin, 1992.

[BK] L. Block, J. Keesling, A characterization of adding machine maps, Topology Appl. 140 (2004) $151-161$.

[BKM] L. Block, J. Keesling, M. Misurewicz, Strange adding machines, Ergodic Theory Dynam. Systems 26 (2006) 673-682.

[BL] A. Blokh, M. Lyubich, Measurable dynamics of S-unimodal maps of the interval, Ann. Sci. École Norm. Sup. (4) 24 (1991) 545-573.

[BM1] A. Blokh, M. Misiurewicz, Wild attractors of polymodal negative Schwarzian maps, Comm. Math. Phys. 199 (1998) 397-416.

[BM2] A. Blokh, M. Misiurewicz, Typical limit sets of critical points for smooth interval maps, Ergodic Theory Dynam. Systems 20 (2000) 15-45. Erratum in Ergodic Theory Dynam. Systems 23 (2003) 661.

[BH] A. M. Bruckner, T. Hu, On scrambled sets for chaotic functions, Trans. Amer. Math. Soc. 301 (1987) 289-297.

[Br1] H. Bruin. Invariant measures of interval maps, Ph.D. Thesis, University of Delft, 1994.

[Br2] H. Bruin, Combinatorics of the kneading map, Internat. J. Bifur. Chaos Appl. Sci. Engrg. 5 (1995) 1339-1349. 
[Br3] H. Bruin, Topological conditions for the existence of absorbing Cantor sets, Trans. Amer. Math. Soc. 350 (1998) 2229-2263.

[Br4] H. Bruin, (Non)invertibility of Fibonacci-like unimodal maps restricted to their critical omegalimit sets, Preprint 2008.

[BHa] H. Bruin, J. Hawkins, Exactness and maximal automorphic factors of unimodal interval maps, Ergodic Theory Dynam. Systems 21 (2001) 1009-1034.

[BKNS] H. Bruin, G. Keller, T. Nowicki, S. van Strien, Wild Cantor Attractors exist, Ann. of Math. (2) 143 (1996) 97-130.

[BKS] H. Bruin, G. Keller, M. St.-Pierre, Adding machines and wild attractors, Ergodic Theory Dynam. Systems 17 (1997) 1267-1287.

[BSS] H. Bruin, W. Shen, S. van Strien, Existence of unique SRB-measures is typical for real unicritical polynomial families, Ann. Sci. École Norm. Sup. (4) 39 (2006) 381-414.

[BRSS] H. Bruin, J. Rivera-Letelier, W. Shen, S. van Strien, Large derivatives, backward contraction and invariant densities for interval maps, Invent. Math. 172 (2008) 509-533.

[CL] H. Cai, S. Li, Distortion of interval maps and applications, Nonlinearity (to appear).

[D] R. H. Day, Complex economic dynamics. Vol. I. An introduction to dynamical systems and market mechanisms, MIT Press, Cambridge, 1994.

[E] R. Engelking, Dimension theory, PWN-Polish Scientific Publishers, Warsaw, 1978.

[G] T. Gedeon, There are no chaotic mappings with residual scrambled sets, Bull. Austral. Math. Soc. 36 (1987) 411-416.

[GKR] C. Good, R. Knight, B. Raines, Nonhyperbolic one-dimensional invariant sets with a countably infinite collection of inhomogeneities, Fund. Math. 192 (2006) 267-289.

[GLT] P. J. Grabner, P. Liardet, R. F. Tichy, Odometers and systems of enumeration, Acta Arith. 70 (1995) 103-125.

[GSS] J. Graczyk, D. Sands, G. Świątek, Metric attractors for smooth unimodal maps, Ann. of Math.(2) 159 (2004) 725-740.

[Gu] J. Guckenheimer, Sensitive dependence to initial conditions for one-dimensional maps, Comm. Math. Phys. 70 (1979) 133-160.

[HS] J. Hawkins, C. Silva, Characterizing mildly mixing actions by orbit equivalence of products, New York J. Math, 3A (1998) 99-115.

$[\mathrm{H}] \quad$ F. Hofbauer, The topological entropy of a transformation $x \mapsto a x(1-x)$, Monatsh. Math. 90 (1980) 117-141.

[HK] F. Hofbauer, G. Keller. Quadratic maps without asymptotic measures, Commun. Math. Phys. 127 (1990) 319-337.

[HY] W. Huang, X. Ye, Homeomorphisms with the whole compacta being scrambled sets, Ergodic Theory Dynam. Systems 21 (2001) 77-91.

[Ja] M. V. Jakobson, Absolutely continuous invariant measures for one-parameter families of onedimensional maps, Commun. Math. Phys. 81 (1981) 39-88.

[JK] K. Janková, J. Smítal, A characterization of chaos, Bull. Austral. Math. Soc. 34 (1986) 283-292.

[J1] V. Jiménez López, $C^{1}$ weakly chaotic functions with zero topological entropy and non-flat critical points, Acta Math. Univ. Comenian. (N.S.) 60 (1991) 195-209.

[J2] V. Jiménez López, Large chaos in smooth functions of zero topological entropy, Bull. Austral. Math. Soc. 46 (1992) 271-285.

[J3] V. Jiménez López, Order and chaos for a class of piecewise linear maps, Internat. J. Bifur. Chaos Appl. Sci. Engrg. 5 (1995) 1379-1394.

[Jo] S. D. Johnson, Singular measures without restrictive intervals, Comm. Math. Phys. 110 (1987) 185-190.

[K] I. Kan, A chaotic function possessing a scrambled set with positive Lebesgue measure, Proc. Amer. Math. Soc. 92 (1984) 45-49.

[Ke] G. Keller. Exponents, attractors and Hopf decompositions for interval maps, Ergodic Theory Dynam. Systems 10 (1990) 717-744.

[Ko] O. S. Kozlovski, Getting rid of the negative Schwarzian derivative condition, Ann. of Math. (2) 152 (2000) 743-762. 
[Le] F. Ledrappier, Some properties of absolutely continuous invariant measures on an interval, Ergodic Theory Dynam. Systems 1 (1981) 77-93.

[LS] S. Li, W. Shen, Hausdorff dimension of Cantor attractors in one-dimensional dynamics, Invent. Math. 171 (2008) 1629-1643.

[LY] T. Li, J. A. Yorke, Period three implies chaos, Amer. Math. Monthly 82 (1975) 985-992.

[Ly1] M. Lyubich, Ergodic theory for smooth one dimensional dynamical systems, Stony Brook preprint $1991 / 11$.

[Ly2] M. Lyubich, Combinatorics, geometry and attractors of quasi-quadratic maps, Ann. of Math. (2) 140 (1994) 347-404.

[LM] M. Lyubich, J. Milnor, The Fibonacci unimodal map, J. Amer. Math. Soc. 6 (1993) $425-457$.

[MMN] M. Majumdar, T. Mitra, K. Nishimura (eds.), Optimization and chaos, Studies in Economic Theory 11, Springer-Verlag, Berlin, 2000.

[M] R. Mañé, Hyperbolicity, sinks and measure in one-dimensional dynamics, Comm. Math. Phys. 100 (1985) 495-524. Erratum in Comm. Math. Phys. 112 (1987) 721-724.

[Ma] M. Martens, Interval Dynamics, Ph.D. Thesis, Delft University of Technology, 1990.

[MMS] M. Martens, W. de Melo, S. van Strien, Julia-Fatou-Sullivan theory for real one-dimensional dynamics, Acta Math. 168 (1992) 273-318.

[MT] M. Martens, C. Tresser, Forcing of periodic orbits for interval maps and renormalization of piecewise affine maps, Proc. Amer. Math. Soc. 124 (1996) 2863-2870.

[MS] W. de Melo, S. van Strien, One dimensional dynamics, Ergebnisse Series 25, Springer-Verlag, Berlin, 1993.

[Mi] M. Misiurewicz, Chaos almost everywhere, in: Iteration theory and its functional equations (Lochau, 1984), 125-130, Lecture Notes in Math., 1163, Springer, Berlin, 1985.

[P] J. Piórek, On the generic chaos in dynamical systems, Univ. Iagel. Acta Math. 25 (1985) $293-$ 298.

[S1] J. Smítal, A chaotic function with some extremal properties, Proc. Amer. Math. Soc. 87 (1983) 54-56.

[S2] J. Smítal, A chaotic function with a scrambled set of positive Lebesgue measure, Proc. Amer. Math. Soc. 92 (1984) 50-54.

[S3] J. Smítal, Chaotic functions with zero topological entropy, Trans. Amer. Math. Soc. 297 (1986) 269-282.

[SS] J. Smítal, M. Štefánková, Omega-chaos almost everywhere, Discrete Contin. Dyn. Syst. 9 (2003) $1323-1327$.

[St] E. Straube, On the existence of invariant, absolutely continuous measures, Comm. Math. Phys. 81 (1981) 27-30.

[vS] S. van Strien, Transitive maps which are not ergodic with respect to Lebesgue measure, Ergodic Theory Dynam. Systems 16 (1996) 833-848.

[SV] S. van Strien, E. Vargas, Real bounds, ergodicity and negative Schwarzian for multimodal maps, J. Amer. Math. Soc. 17 (2004) 749-782. Erratum in J. Amer. Math. Soc. 20 (2007) 267-268.

[Y] L.-S. Young, Recurrence times and rates of mixing, Israel. J. Math. 110 (1999) 153-188.

Department of Mathematics

University of Surrey

Guildford, Surrey, GU2 7XH

UK

h.bruin@surrey.ac.uk

http://personal .maths.surrey.ac.uk/st/H.Bruin/

Departamento de Matemáticas

Universidad de Murcia

Campus de Espinardo, 30100 Murcia

Spain 
vjimenez@um.es

http://www.um.es/docencia/vjimenez 